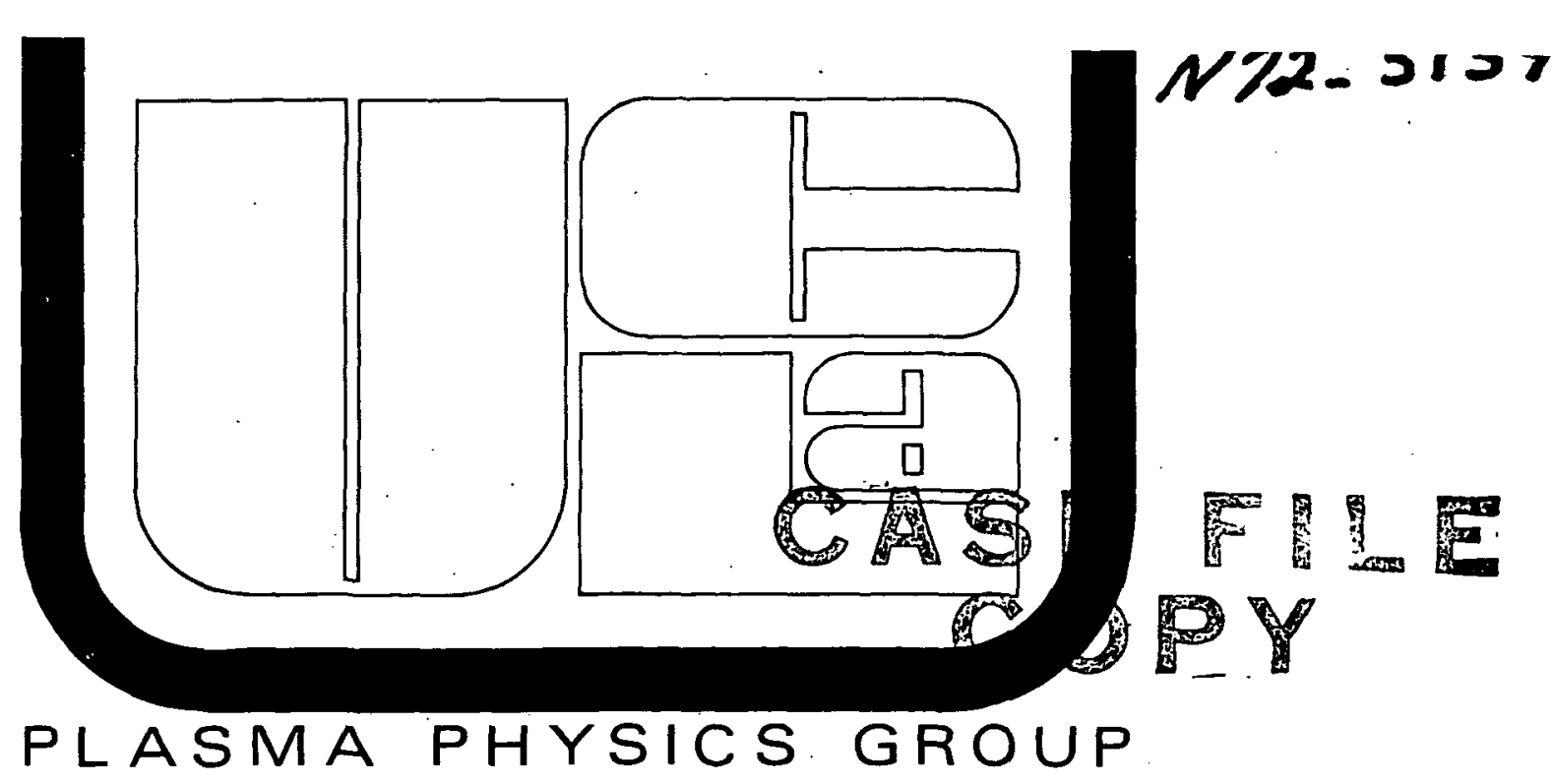

MAGNETOSPHERIC ELECTRONS

by

F. V. Coroniti ${ }^{1}$ and R. M. Thorne ${ }^{2}$

July, 1972

PPG-123

UNIVERSITY OF CALIFORNIA LOS ANGELES

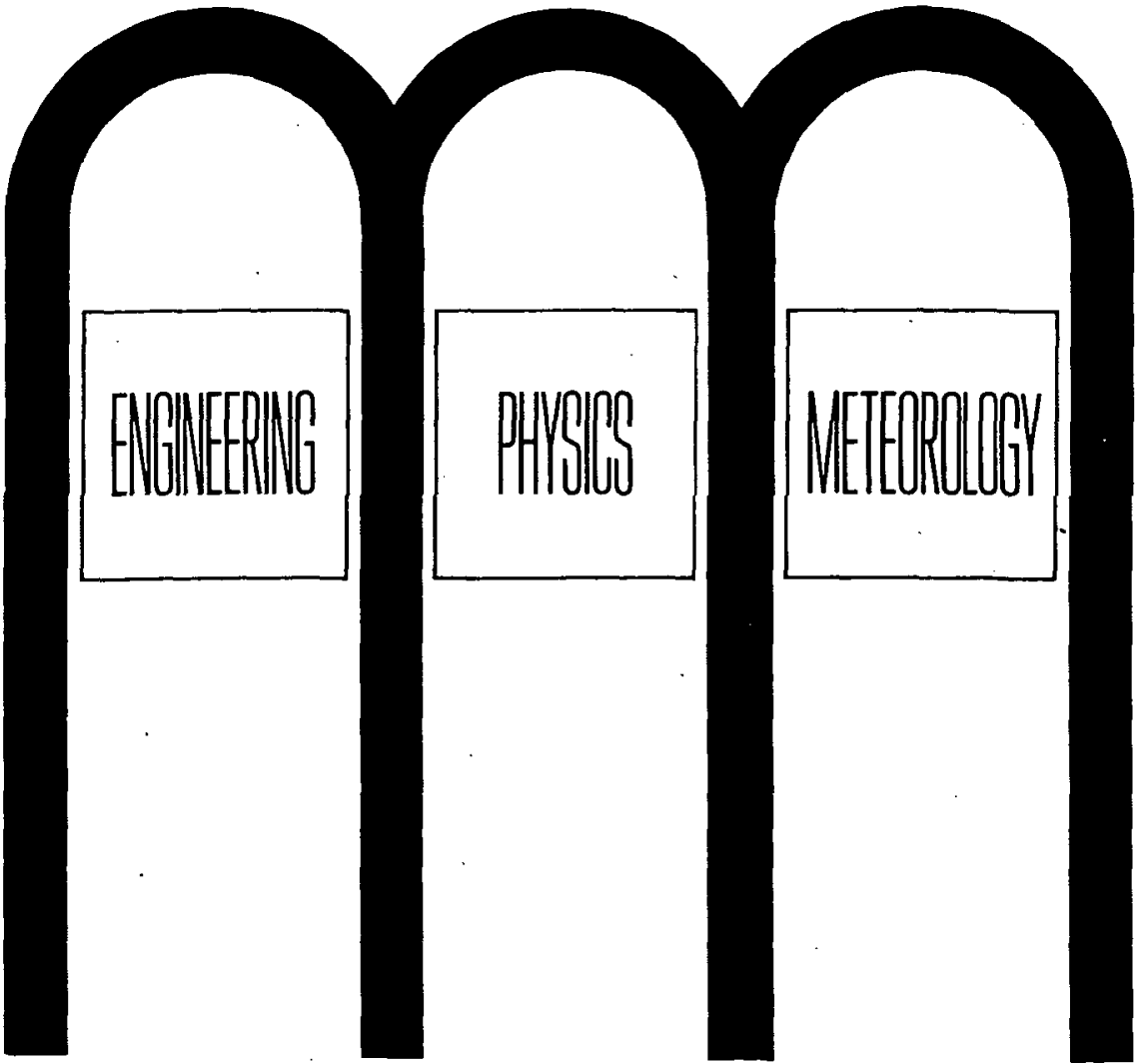




\section{MAGNETOSPHERIC ELECTRONS}

by

F. V. Coroniti ${ }^{1}$ and R. M. Thorne ${ }^{2}$

July, 1972

PPG -123

\footnotetext{
$1_{\text {Physics Department, University of California, Los Angeles }}$ Los Angeles, California 90024
}

\footnotetext{
${ }^{2}$ Meteorology Department, University of Caiifornia, Los Angeles Los Angeles, California 90024
} 


\section{1.) INTRODUCTION}

The satellite era of magnetospheric physics began with the discovery of the electron Van Allen radiation belts. By the late sixties numerous satellite surveys had determined the energy spectra, spatial distribution, and temporal variations of electrons with energies between $0.1 \mathrm{KeV}-1 \mathrm{MeV}$. During this period the development of hydromagentic models of the magnetosphere and the application of. plasma turbulence theory resulted in a rough quantitative understanding of electron dynamics. The purpose of this review is to sumarize the present theoretical understanding of magnetospheric electrons. Such a review will inevitably neglect many important observational features which are not yet understood, and some of the theoretica1. concepts may not always correspond to waht is actually observed. Finally, we apologize in advance to any theorist whose work is not discussed or referenced.

First let's define our reference system, i.e., structure of the magnetosphere. Figure 1 shows the magnetosphere's magnetic field topology separated into several distinct regions. The post-shock solar wind flow confines the geomagnetic field to a roughly 10 earth radii $\left(R_{E}\right)$ hemispherical cavity on the dayside. The resistive annihilation of magnetic fields at neutral lines or points on the dayside magnetopause results in the direct connection of interplanetary and geomagnetic field lines: Thus the solar wind flow over the magnetosphere exerts a tangential Maxwell stress which pulls the geomagnetic field out into a long tail extending many hundreds of $R_{E}$ downstream on the nightside. Lines of force in the high latitude lobes of the tail are open to the solar wind, while lines in the plasma sheet have reconnected with their dipole partiners, and hence are closed. The plasma sheet carries the self-consistent currents required to form the opposed magnetic fields of the tail. Earthward from the tail is a quasi-dipolar region whose field lines leave and enter the earth at latitudes corresponding to the statistical auroral zone (1). Further inward is a region of dense, cold plasma, called the plasmasphere, which is a diffusive extension of the ionosphere. The classical outer Van Allen belt is typically located outside the plasmapause (62, 64), while the inner zone occurs well within the plasmasphere.

Theoretically, the structure of the earth's radiation belts can be divided into three interrelated problems: (1) determination of the source of low energy electrons; (2) determination of how electrons are transported from their source; (3) evaluation of possible sinks of electrons from the magnetospheric system. The evaluation of electron sinks is most directly amenable to plasma physical theory and relates to the laboratory confinement of thermonuclear plasmas; hence our understanding of sinks is quite extensive. The transport problem primarily concerns the hydromagnetic structure and dynamics of the magnetosphere. Qualitatively, transport is well understood, but accurate quantitative models are years away from development. Surprisingly, the source of magnetospheric electrons is probably. the least understood of the three problems.

The largest available volume of energetic plasma is the tail plasma sheet. The solar wind probably penetrates directly into the plasma sheet at a weak field region near the center, but whether the region of dominant entry is near the earth or in the distant tail is still unresolved. The open lobes of the tail might also contain very low energy plasma which is as yet undetected. This plasma could be accelerated to plasma sheet energies by turbulence within the sheet itself or possibly by the magnetic field annihilation process. In addition to the plasma sheet, the polar cusp $(34,37)$ and the polar wind flow of ionospheric plasma into the magnetosphere (7) might be additional, though limited, sources of plasma.

The transport of electrons from the plasma sheet to the auroral zone is accomplished by internal magnetospheric convection. Dungey (25), Petschek (57), Levy et al. (47), and Axford et al. (5) have argued that when the solar magnetic field has a southward component, direct merging or connection of interplanetary and geomagnetic fields establishes a convective flow of magnetic flux into the tail. 
Flux conservation requires a second neutral line in the plasma sheet and a convective return flow of plasma sheet particles and flux toward the nightside. Although. the original convection models were steady state, the most intense convection occurs for relatively short times, the order of 30 minutes, during the expansion phase of magnetospheric substorms (1). Thus the transport rate of electrons from the plasma sheet to the auroral zone is keyed to substorm activity.

If convection were steady and electron dynamics were purely adiabatic, convection would eventually flow plasma sheet electrons to the dayside magnetopause where they would return to the solar wind. However, sporadic intensification and subsidence of convection often injects large electron fluxes into the auroral zone whereupon they execute guiding center drift motion around the earth (54). Earthward transport of electrons, however, will not cease with convection. An electron's drift adiabatic invariant can be violated by resonant interactions with electromagnetic fluctuations whose frequency matches a harmonic of the electron's drift frequency. A net radial or L-shell (51) displacement results, and the electron may diffuse inward to lower L-shells (61).: Since low energy ( $<40 \mathrm{KeV}$ ) electrons have drift periods longer than either the time between substorms or their precipitation lifetimes (43), inward radial diffusion constitutes an effective transport process for only high energy electrons.

As a magnetic flux tube flows from the deep tail toward the earth, its volume decreases. Plasma on these flowing field lines is more or less adiabatically compressed, and thus arrives in the auroral zone with a higher density and temperature; the energy increase originates from the convection electric field. Maximum convection potentials taken across the magnetosphere can be as large as 100-300 KeV during substorms (35). However, since such potentials typically persist for only a small fraction of the time required to drift across the full potential drop, electrons may only gain some $10^{\prime} \mathrm{s}$ of $\mathrm{KeV}$ energy due to convection. After convective injection into the auroral zone, radial diffusion may transport electrons into regions of high magnetic field strength. Since radial diffusion approximately conserves the first and second adiabatic invariants (61), electrons gain energy as they diffuse inward at the expense of the fluctuating electromagnetic fields driving the diffusion. Energies of some $100^{\prime} \mathrm{s}$ of $\mathrm{KeV}$ are attainable, so that inner Van Allen belt electrons are usually more energetic than are auroral electrons.

The ultimate fate of a plasma sheet electron is not controlled by convection or radial diffusion alone. Since both convection and radial diffusion tend to heat electrons preferentially in the direction perpendicular to the magnetic field, a pitch-angle anisotropy eventually develops in the electron distribution function during transport into the dipole magnetic field. (The pitch-angle $\alpha=\tan ^{-1}\left(v_{1} / v_{11}\right)$, where $v_{1}\left(v_{11}\right)$ is the electron velocity perpendicular (parallel) to the magnetic field.) An additional anisotropy is always present due to the mirror-loss cone property of the geomagnetic field; electrons with small pitch angles at the magnetic equator can reach the lower ionosphere where collisions with neutrals remove them from the system. Since the maximum entropy state of any gas corresponds to an isotropic pitch-angle distribution, anisotropy is a source of free energy which is available to destabilize various modes of plasma turbulence. Electrons relinquish energy to the plasma waves and in the process are scattered by the wave electromagnetic fields to smaller pitch angles, i.e., toward the loss cone. Hence plasma turbulence can significantly reduce the fluxes of electrons wherever the loss cone is large and whenever the fluxes and anisotropies are sufficiently large to be unstable $(41,17)$.

In Section 2, we discuss low energy auroral electrons (0.1-10.0 KeV). The macroscopic characteristics of these electrons are determined by convective transport and precipitation sinks. Section 3 considers the higher energy $(>40 \mathrm{KeV})$ Van Allen electrons. Their transport to deep inside the plasmasphere is probably controlled by inward radial diffusion. The observed precipitation loss and pitchangle distributions of plasmaspheric Van Allen electrons are now in excellent 
agreement with the theoretical calculations by Lyons et al. (50).

\section{0) CONVECTION DOMINATED ELECTRONS IN THE AURORAL ZONE}

In Section 2.1, we compare plasma sheet electron spectra with those observed in the auroral zone and argue that the auroral spectra are consistent with inward convection. Section 2.2 considers the spatial distribution calculated assuming only convective and adiabatic guiding center transport. The observed spatial distribution of auroral electrons is then reviewed in Section 2.3. In Section 2.4 non-adiabatic plasma turbulence precipitation sinks are added to the convection spatial distribution model.

\section{1) The Plasma Sheet as the Source of Auroral Electrons}

Figure 2a, b shows the differential energy spectra of plasma sheet electrons observed by Hones et a1. (39) during quiet geomagnetic conditions (2a) and following the expansion phase of a substorm (2b). Both electron spectra are approximately Maxwellian between the energies of 0.1 to $10.0 \mathrm{KeV}$, with the quiet (substorm) spectra having a mean energy of $0.42 \mathrm{KeV} .(2.6 \mathrm{KeV})$ and an estimated total density of 0.5 (0.2) particles $/ \mathrm{cm}^{3}$. Electron measurements at $40 \mathrm{KeV}$, however, show fluxes considerably in excess of those expected for a Maxwellian distribution thus indicating an extensive high energy tail (32). Although the electron distribution is generally isotropic $(6,38)$, occasionally very intense "beams" of electrons are observed which are thought to be guided along the tail magnetic field lines (39).

Figure 3 curve $I$ is a plot of the differential energy flux for pitch angles near the loss cone obtained by DeForest and McIlwain (24) on the synchronous saté1lite ATS-5 near midnight prior to a substorm. The spectrum is roughly Maxwellian between 0.05 and $10 \mathrm{KeV}$, with a mean energy of $2.5 \mathrm{KeV}$ and total density of $5.5 \mathrm{~cm}^{-3}$. The simultaneously detected perpendicular flux (not shown but virtually identical to curve I) was essentially equal to the parallel flux so that the anisotropy was either zero or smal1. Curves II, III, and IV demonstrate the variability of the auroral electron spectra.

If we assume that convection approximately conserves the first two adiabatic invariants, then the electron distribution observed by Vela at $18 R_{E}$ in the tail can be extrapolated inward to $6 R_{E}$ (roughly. ATS 5). For typical field strengths we take $B\left(18 R_{E}\right) \approx 10^{-4}$ Gauss; and $B\left(6 R_{E}\right) \approx 10^{-3}$ Gauss; the length of the Vela field line might be $\ell_{11}\left(18 \mathrm{R}_{\mathrm{E}}\right) \approx 40 \mathrm{R}_{\mathrm{E}}$ while at $6 \mathrm{R}_{\mathrm{E}} \ell_{11}\left(6 \mathrm{R}_{\mathrm{E}}\right) \approx 20 \mathrm{R}_{\mathrm{E}}$. From the data of Figure 2, conservation of the first adiabatic invariant would give a

perpendicular temperature at $6 R_{E}$ of $T_{\perp}\left(6 R_{E}\right) \approx T_{\perp}\left(18 R_{E}\right)\left(\frac{B\left(6 R_{E}\right)}{B\left(18 R_{E}\right)}\right) \approx 2-4 \mathrm{KeV}$.

Conservation of the second invariant yields a parallel temperature at $6 R_{E}$ of $T_{11}\left(6 R_{E}\right) \approx T_{11}\left(18 R_{E}\right)\left[\ell_{11}\left(18 R_{E}\right) / \ell_{11}\left(6 R_{E}\right)\right]^{2} \approx 0.8-1.6 \mathrm{KeV}$. The resultant thermal anisotropy at $6 R_{E}$ is $A=T_{1} / T_{11}-1 \approx 1.5$. The density is inversely proportional to the flux tube volume which scales as $\ell_{11} / B$, so that at $6 R_{E}$ we have $n\left(6 R_{E}\right)=$ $n\left(18 R_{E}\right)\left[\frac{\ell_{11}\left(18 R_{E}\right)}{B\left(18 R_{E}\right)} \frac{B\left(6 R_{E}\right)}{\ell_{11}\left(6 R_{E}\right)}\right] \approx 6 \mathrm{~cm}^{-3}$. These estimates are extremely crude since the adiabatic invariants are to some extent probably violated by plasma turbulence and magnetic inhomogeneities on gyro-radius scale lengths. However, the general agreement of the scaled and measured (Figure 3) parameters clearly indicates that convection from the plasma sheet is the dominant source and transport process for the auroral electrons. 


\section{2) Convection Electron Spatial Distribution}

If the magnetospheric electric and magnetic field configurations were known, the adiabatic guiding center equations of motion could be solved to determine the electron orbits and thus the electron velocity and spatial distributions. Lacking this detailed knowledge several authors $(9,67)$ have employed model fields to carry out the calculations. Typical of these results is the work of Kavanagh et al. (40) which is shown in Figure 4. These authors specifically assume that electrons at $10 \mathrm{R}_{\mathrm{E}}$ behind the earth have an energy of $1 \mathrm{KeV}$ in a magnetic field of $2 \times 10^{-4}$ Gauss. Furthermore, the electrons are restrained to mirror in the equatorial plane, the plasmapause is taken as a perfectly conducting sphere located at $4 \mathrm{RE}$, a $50 \mathrm{KV}$ convection electric potential is adopted, and any charge separation and ionospheric polarizat on electric fields are neglected. The assumption of $90^{\circ}$ pitch angle at the equator implies that the drift trajectory is along contours of constant to total energy $W=q \phi+\underline{\mu} \cdot B$. where $\phi$ is the electric potential, and $\underline{\mu}$. is the first adiabatic invariant assumed equal to $5 \mathrm{MeV} /$ Gauss. A11 non-adiabatic processes are neglected.

Electrons which start on the morning side of the tail flow rapidly around local dawn and out to the magnetopause taking about four hours for the trip. The relatively high drift speed implies that the density of these electrons remains low. Electrons starting in the pre-midnight region, except those very close to the dusk magnetopause, encounter a forbidden region (3) at about $8 \mathrm{R}_{\mathrm{E}}$, inside of which they cannot penetrate without violating energy conservation. Near the forbidden region, called the Alfven layer, the convection electric field drift is approximately balanced by the magnetic gradient, curvature, and corotation electric field drifts. Pre-midnight, the electrons spend about 3 to 6 hours in the vicinity of the Alfven layer, and then drift more rapidly around toward morning. The electron density maximizes near the dusk Alfven layer since the drift speed is slowest there, and the electron thermal energy maximizes near the dawn Alfven layer where the largest magnetic field strength is attained. Thus this model predicts that intense fluxes of 5 to $10 \mathrm{KeV}$ electrons should be detected between about 5 and $7 \mathrm{RE}$ starting at 0300 time and continuing eastward to about local noon. Also note that a second region of very dense 3 to $5 \mathrm{KeV}$ electrons should occur near $8 \mathrm{R}_{\mathrm{E}}$ at about 2000 local time. Finally, if a spectrum of electron energies is present in the plasma sheet, the convection-drift model predicts that the lower energy electrons would penetrate further into the dipole than the higher energy electrons.

\section{3) Observed Electron Spatial Distribution}

A sharp inner edge to the plasma sheet (2 KeV) electrons was first detected by Vasyliunas $(71,72)$ on OGO's 1 and 3 at about $10 R_{E}$ in the dusk-midnight region. Figure 5, from Schield and Frank (65), shows a detailed radial profile of the plasma sheet inner edge near local midnight for electrons between 0.1 and $50.0 \mathrm{KeV}$. The flux in the higher energy channel ( 5 to $24 \mathrm{KeV}$ ) begins decreasing around $7 \mathrm{R}_{\mathrm{E}}$ while the lower energy electrons $(0.5$ to $5.0 \mathrm{KeV})$ penetrate to about $6.4 \mathrm{R}_{\mathrm{E}}$ before decreasing. The spatial scale length of the flux gradient is about $0.5 \mathrm{RE}$. Across the inner edge the integrated energy density of 0.7 to $50.0 \mathrm{KeV}$ remained constant until the plasmapause was encountered at $5.9 \mathrm{R}_{\mathrm{E}}$, thus indicating that the density loss of $>0.7 \mathrm{KeV}$ electrons was compensated by a density gain in the low energy 0.09 to $0.7 \mathrm{KeV}$ electrons (see also Vasyliunas, 71,72 ).

The azimuthal structure of the inner edge has been mapped by Vasyliunas (72). In the dusk-midnight region the boundary is sharp and varies in radial distance from 7 to $8 R_{E}$ at midnight; to 10 to $11 R_{E}$ at dusk, and finally merges with the magnetopause near local noon. On the morning-side the inner edge is also between 8 and $10 R_{E}$, but is less sharply defined with appreciable KeV electron fluxes extending inward to the plasmapause. However, the most intense fluxes, of order $10^{9}-10^{10}$ electron $/ \mathrm{cm}^{2}$-sec from 0.1 to $2.0 \mathrm{KeV}$, exist beyond $8 \mathrm{RE}$. On Injun $V$, Frank and Ackerson (33) have detected these electrons as an almost uniform electron flux 
between 0.1 and $10 \mathrm{KeV}$ extending from $68^{\circ}$ to $78^{\circ}$ invariant altitude at $0900-1000$ local. time. Finally, at synchronous altitude (6.6 $\left.\mathrm{R}_{\mathrm{R}}\right)$, DeForest and McIlwain (24) report that the typical (see Table I) electron density, flux, and energy density maximize in the midnight to 0300 local time sector, and then fall off to a minimum about 1800 local time.

Now let's compare the observed and theoretical spatial distributions. In the dusk-midnight region the Alfven layer roughly coincides with the inner edge of the plasma sheet. Furthermore, the more earthward penetration of lower energy electrons agrees qualitatively with Alfven layer theory. However, at dawn the theoretically expected high density of 5-10 KeV fluxes between 5 and $7 \mathrm{R}_{\mathrm{E}}$ is not generally observed $(24,33)$. In the noon-dusk region, the plasma sheet boundary is just inside the magnetopause, which disagrees with the expected Alfven layer at $8 \mathrm{R}_{\mathrm{E}}$. Finally, the constancy of the electron density across the inner edge is, at best, difficult to explain on the basis of the convection-Alfven layer theory. We conclude that while convection roughly accounts for the electron spatial distribution on the nightside, additional physics must be added to explain the observations, especially at dawn.

\section{4) Coupling of Convection and Precipitation}

Petschek and Kennel (58) first predicted that first adiabatic invariant violations by pitch-angle scattering interactions with high frequency wave turbulence would diffuse inward convecting electrons into the atmospheric loss cone and produce an inner edge to the electron plasma sheet fluxes via precipitation. Two modes of plasma turbulence are observed on nightside auroral lines of force: 1) the electromagnetic whistler mode with the frequencies below the electron cyclotron frequency $(10) ; 2$ ) an electrostatic mode with frequencies between 1.25 and 1.75 of the electron cyclotron frequency (44). Both modes are destabilized by a pitch-angle or loss-cone anisotropy with $T_{1} / T_{1}>1(4,41,79)$ as is produced by the inward convection flow.

In general, the electron precipitation rate and lifetime are determined by the amplitude of the wave turbulence. However, when the wave turbulence level is sufficiently strong to maintain pitch-angle isotropy across the equatorial loss cone, the precipitation lifetime depends only on the geometric probability of an electron being in the loss cone and the electron escape time from the system (43). For a dipole field this minimum electron or strong diffusion lifetime is given by $T_{\min } \propto L^{4} / \sqrt{E(\mathrm{KeV})}$, where $E$ is the electron energy in $\mathrm{KeV}$. For $L=8$, and $\mathrm{E} \approx 1 \mathrm{KeV}, \mathrm{T}_{\min } \approx 2 \times 10^{3} \mathrm{sec}$, which is considerably shorter than the 3 to 6 hour electron drift time through the dusk-midnight region given by convection-Alfven layer theory. Hence, even if the precipitation lifetime somewhat exceeds the strong diffusion $T_{m i n}$, very few convection electrons in the vicinity of the Alfven-layer would survive to drift around to local dawn. Since $T_{\min }$ decreases with increasing energy, the lower energy electrons penetrate further into the dipole before being $10 s t$.

Vasyliunas (see Kennel, (43)) has incorporated precipitation losses into a convection-guiding center drift model for the electron spatial distribution. Furthermore, since the proton precipitation flux, even on strong diffusion, is usually an order of magnitude smaller than the electron precipitation flux, the maintenance of charge neutrality in space requires that cold ionospheric electrons flow out into the magnetosphere to replace the precipitated electrons (45).

Vasyliunas also permits these ionospheric electrons to thermalize with the convection electrons in less than a flow time. His basic results (see Figure 4) are: a) the calculated location of the plasma sheet inner edge or temperature gradient boundary roughly agrees with observations; b) the electron density is constant across the inner edge due to the charge neutralizing ionospheric electrons; c) the electron density and mean energy between 5 and $8 . R_{E}$ at local dawn are low due to the precipitation loss of the energetic convection electrons and their replacement by cooler ionospheric electrons; d) outside $8 R_{E}$ at dawn the long 
minimum lifetime results in the relatively high electron density and mean energy; e) few electrons survive to drift into the noon-dusk region. Hence the coupling of convection and precipitation produces an electron spatial distribution which is in much better agreement with observations than that predicted by the convectionAlfven layer theory alone.

\section{0) GEOMAGNETICALLY TRAPPED ELECTRONS}

Although auroral electrons ( $>>6$ ) rarely survive for longer than several hours in the radiation belts, higher energy $(>40 \mathrm{KeV})$ electrons can be quasi-stably trapped in the region $L \lesssim 6$ for many days or even years. In Section 3.1, we discuss possible sources of these Van Allen electrons. Section 3.2 briefly considers the inward transport of electrons to low L-shells by radial diffusion. The geomagnetically quiet and disturbed electron spatial structure is reviewed in Section 3.3 . Section 3.4 considers the precipitation loss of energetic electrons due to whistler turbulence inside the plasmasphere, and Section 3.5 discusses the rapid loss of $>\mathrm{MeV}$ electrons due to interactions with ion cyclotron turbulence at the plasmapause.

\section{1) Source of Van Allen Electrons}

The sporadic enhancements of inward convection which occur during magnetospheric substorms not only increase auroral electron fluxes, but also produce an injection and acceleration of $>40 \mathrm{KeV}$ electrons. Figure 6 (56) shows the flux increase of 50 to $10^{3} \mathrm{KeV}$ electrons observed on ATS-1 which occurred within minutes of the expansion phase onset of a substorm (indicated by the sharp negative change in the $\mathrm{H}$-component of the College magnetogram). A1though they have been detected at a11 local times, these substorm enhancements are largest near midnight and are limited to the region exterior to the stormtime plasmapause (77).

Since the total convection potential across the magnetosphere rarely exceeds 100-200 KV (14), the substorm enhancement of MeV electrons is not explicable by adiabatic compression alone. A possible non-adiabatic heating process is the stochastic acceleration of a few higher energy electrons which can interact with the intense plasma wave turbulence generated by auroral electrons (43). Electrons might also become trapped in the electrostatic potential well of a single wave and be accelerated to high energy if the wave phase speed increased $(46,66)$. However; neither of these processes has been evaluated in detail, and acceleration mechanisms remain virgin territory, theoretically.

\section{2) Inward Radial Diffusive Transport}

Since substorm acceleration and injection processes can prime the outer radiation belts with energetic electrons, the next problem is to transport these electrons to lower L-shells. For trapped electrons, a steady convection electric field would not produce any inward transport, but only a distortion of the guiding center orbit. However temporal variations in the convection or possibly wave electric field with time scales comparable to the electron azimuthal drift period can result in a net inward L-shell displacement. If these time variations are random, and conserve the first two adiabatic invariants (54), this spatial transport is described by a diffusion equation in L-space $(8,27,61)$. The diffusion coefficient is given by

$$
D_{L L}=\frac{c^{2} L^{6}}{8 B_{0}^{2}}[P(\nu)]_{\nu=\tau_{D}}-1
$$

where $B_{0}$ is the $L=1$ equatorial dipole field strength, $P(\nu)$ is the electric field power spectral density at frequency $\nu, \tau_{D}=\frac{2 \pi e R_{E}{ }^{2}}{3 \mu c}\left\{1+\frac{2 \mu B_{0}}{m c^{2}}\right\}^{1 / 2}$ is the azimuthal drift period, $c$ is the speed of light, $e$ is the electronic charge, and $m$ is the electron rest mass. 
Cornwall $(18,20)$ has evaluated $D_{L L}$ for substorm-like electric field variations which rise rapidly and then decay exponentially with a time constant $\mathrm{T} / 2$; he finds

$$
D_{L L}=\frac{c^{2}\left\langle E^{2}\right\rangle}{4 B_{0}^{2}} L^{6}\left[\frac{T}{1+\left(\frac{\omega_{D} T}{2}\right)^{2}}\right]
$$

where $\left\langle E^{2}\right\rangle$ is the mean-square fluctuating electric field and $\omega_{D}=2 \pi / \tau_{D}$. Low energy electrons with $\omega_{D}^{T} \ll 1$ have $D_{L L} \sim L^{6}$, while ultra-relativistic electrons $\left(\omega_{D} T \gg>1\right)$ have $D_{L L} \sim L_{7}$. Tomassian et al. (70), have used (3.2) with $<E^{2}>1 / 2 \sim$ $0.1-0.3 \mathrm{millivolts} / \mathrm{meter}$ and $\mathrm{T} \sim 1 / 2$ hour to fit the observed inward radial diffusion of inner zone relativistic electrons. These electric fields are comparable to those measured by Mozer (52) and Carpenter et al. (12), and the time scale is consistent with convection enhancements and decay times (22).

\section{3) Spatial Structure of Van Allen Electrons}

With the radial diffusion coefficient reasonably well determined, the L-she11 distribution of electrons could be obtained by simply solving the diffusion equation with a source term at auroral L values. In the absence of sinks, the solution would predict a monotonically increasing electron flux with decreasing L-shell. Typical equatorial electron flux profiles measured by Pfitzer et al. (59) are shown in Figure 7. For quiet conditions (Sept. 20) electrons are distributed into two distinct zones separated by a broad region, referred to as the slot, in wich the fluxes fall below detection threshold. Below $300 \mathrm{KeV}$ the location of the outer zone flux maximum is roughly independent of energy and is correlated with the position of the plasmapause (62); above an MeV the fluxes peak just inside the plasmapause and evince a systematic inward displacement with increasing energy (74). During moderate geomagnetic activity (Sept. 29) lower energy ( $<700 \mathrm{KeV}$ ) electrons are injected to partially fill the slot while the higher energy $(>700 \mathrm{KeV})$ and inner zone $(\mathrm{L}<2)$ electron distributions remain essentially unaffected. Following major magnetic storms, however, substantial flux enhancements occur even in the inner zone $(60,70)$, which then decay on a time scale of many months (78). Hence the slot cannot result from radial diffusive transport alone, and the decay of the storm injected fluxes indicates that rapid sinks of Van Allen electrons exist within the plasmasphere.

Evidence for a strong source of electrons just outside the plasmapause is contained in Figure 8 (Owens and Frank, 55) which shows a two month history of outer zone electron fluxes. During magnetic storms lower energy $(<1.6 \mathrm{MeV})$ electron.fluxes are greatly enhanced $(23,30,31,77)$ and move in unison with the storm-time plasmapause to lower L-values (64). This behavior is consistent with impulsive convective injection to the vicinity of the compressed plasmapause (L $\sim 3$ to 4) $(11,15)$; thus convection provides the outer-zone source for radial diffusive transport into the slot and inner-zone. Also evident in Figure 8 is the rapid depletion of $>1.6 \mathrm{MeV}$ electrons at $L=3$ to 5 during the early phase of a magnetic storm $(28,29,67)$. Vampola (71) has reported precipitation of $1.5 \mathrm{MeV}$ electrons near the plasmapause with roughly isotropic pitch-angle distributions, thus indicating that these electrons are lost due to strong plasma turbulent pitch-angle diffusion. These electrons immediately return after the storm with flux levels exceeding pre-storm values. Hence a strong acceleration source of $\mathrm{MeV}$ electrons must operate throughout the outer zone (see Figure 6). 


\section{4) Electron Slot Formation}

The decay of electrons injected into the slot during storms indicates the preferential loss or precipitation of electrons in this region. Early whistler turbulent-precipitation theories stressed that the unstable, and hence precipitating, electrons must self-consistently generate the pitch-angle diffusing plasma turbulence locally on each flux tube $(4,17,41)$. This self-consistency requirement was stated in terms of a stable trapping flux limit; i.e., fluxes below a threshold value could not sustain a steady turbulence level against wave propagation losses. For the losses of $>100 \mathrm{KeV}$ electrons in the slot, this concept is inapplicable since the electron fluxes are too small to be unstable. However, whistlermode turbulence with frequencies centered at a few hundred $\mathrm{Hz}$ (69) and wave amplitudes of 5 to $50 \mathrm{milligammas}$ is continuously present throughout the slot (inside the plasmapause). At these low frequencies, whistler waves readily propagate across magnetic field lines $(48,53)$ and thus fill the entire slot region. The waves are probably generated by low energy (20-40 KeV) electrons which have diffused to just inside the plasmapause.

Even though stable to whistler emission, $>100 \mathrm{KeV}$ slot electrons can be parasitically scattered by this background whistler turbulence and thus diffuse into the loss cone (42). Since the whistlers propagate obliquely to the magnetic field, electron resonance and diffusion will occur at each harmonic of the electron cyclotron frequency and at Landau resonance (49). Lyons et a1. (50) modeled the whistler hiss spectrum by a Gaussian wave distribution centered at $600 \mathrm{~Hz}$ with a $300 \mathrm{~Hz}$ e-folding width, a relatively isotropic distribution of wave energy in $\mathrm{k}$-space, and a wave intensity which is independent of geomagnetic latitude and L-shell. They then calculated the pitch-angle diffusion coefficient (see Figure 9) averaged over the electron bounce period and summed for all possible resonant interactions. Equatorially mirroring electrons diffuse toward lower pitch-angles by Landau resonance and are subsequently scattered into the loss cone by cyclotron resonance. For low energy electrons ( $20 \mathrm{KeV})$ first order cyclotron resonance dominates the diffusion. Higher energy electrons experience a combination of first order cyclotron resonance near their mirror points plus high harmonic resonance near the geomagnetic equator.

For times during which no strong sources occur in the slot, the pitch-angle diffusion coefficients can be incorporated into the source-free quasilinear diffusion equation $(41,35)$ to solve for the electron pitch-angle distribution and precipitation lifetime (50). A comparison between theoretical pitch-angle distributions and those observed on the 0GO-5 satellite (H. West private communication) is shown in Figure 10. The pronounced lower energy flux enhancements around $90^{\circ}$ result from the slow diffusion rates (which produce steep pitch-angle gradients) in the region of partial overlap between cyclotron and Landau resonance (see Figure 9). At high energies, the region of cyclotron diffusion extends to high pitch-angles and consequently no $90^{\circ}$ enhancements are expected. Theoretical precipitation lifetimes are shown in Figure 11 based on a wide-band wave amplitude of $35 \mathrm{milligammas.} \mathrm{Over} \mathrm{the} \mathrm{energy} \mathrm{range} 0.1-2.0 \mathrm{MeV}$ the predicted lifetimes approximately agree with the observed electron decay rates (Pfitzer; Vampola, private communication). The sharp increase in the electron lifetimes at low. L-values, where electrons no longer interact with the observed whistler turbulence, correctly predicts the location of the slot inner edge. Within the slot region, the lifetimes are relatively short, and any injected electrons are rapidly lost by precipitation. The variation of the lifetimes with energy predicts that the slot's outer edge should occur at higher L-values with decreasing energy, as observed in Figure 7 . However, the precise particle source must be specified in order to solve for the electron structure. 


\section{5) Rapid Loss of Outer-Zone MeV Electrons}

Although the whistler theory is in excellent agreement with the observed electron lifetimes, pitch-angle distributions, and approximate spatial distributions, the rapid loss of outer zone $>1.6 \mathrm{MeV}$ electrons during the storms (Figure 8) requires a much faster loss process than is provided by whistlers. Relativistic electrons ( $>1 \mathrm{MeV})$, however, can also resonate with electromagnetic ion cyclotron waves in the high density regions of the plasmasphere (68). Cornwall et al. (19) have predicted that ring current protons ( $\sim 50 \mathrm{KeV}$ ) would generate intense ion cyclotron turbulence just inside the plasmapause. Thorne and Kennel (68) have shown that parasitic pitch-angle diffusion of relativistic electrons with these waves gives a roughly 50 second precipitation lifetime. Since this lifetime is comparable to the minimum precipitation lifetime, precipitated electrons should be roughly isotropic in pitch-angle, in agreement with recent observations by Vampola (71). Even if rapid acceleration of electrons occurs in the outer zone during storms, as is indicated by the flux enhancements of lower energy electrons $(<1 \mathrm{MeV})$ in Figures 7 and 8 , the parasitic ion cyclotron precipitation is sufficiently fast to keep the relativistic fluxes near background throughout the storm main phase.

\section{0) DISCUSSION}

The coupling of source, transport, and sink processes yields a fairly accurate model for the macroscopic structure and dynamics of magnetospheric electrons. Auroral electrons are controlled by convective transport from a plasma sheet source coupled with a precipitation loss due to whistler and electrostatic plasma turbulence. Outer and inner zone electrons are governed by radial diffusion transport from convection and acceleration sources external to the plasmapause and by parasitic precipitation losses arising from cyclotron and Landau interactions with whistler and ion cyclotron turbulence.

However, several fundamental problems are not yet adequately understood:

- The entry of solar wind plasma into the tail, the heating of this plasma, and the magnetic structure of the plasma sheet must be determined before quantitative models of the basic electron source can be constructed. The solution of these problems involves the plasma physics of magnetic field annihilation and the dynamics of high pressure plasmas, neither of which are currently well-developed.

- The extent to which the polar wind flow of ionospheric plasma into the magnetosphere provides a source of electrons must be evaluated.

-A self-consistent model of magnetospheric convection is needed in order to specify the internal transport rates as a function of the imposed solar wind and ionospheric boundary conditions. Since convection is regulated by substorms and magnetic storms, convection theories must include time dependent changes of the entire magnetospheric configuration (21).

- The interaction between convection and the ionosphere could modify the electron distributions in the magnetosphere. At the plasma sheet inner edge, the spatial gradient in the electron precipitation flux produces an inhomogeneous ionospheric condictivity (22). The convection electric field driven ionospheric currents may require closure paths in the magnetosphere which must be fed via field-aligned currents. If these field-aligned currents become sufficiently intense to generate current-driven plasma turbulence (45), an anomalous resistance and quasi-DC parallel electric field will develop somewhere along the field line. In addition to local Joule heating, a small fraction of the current-carrying electrons would be expected to runaway, i.e., be accelerated to high energies. These runaway electrons may constitute the narrow energy beams observed in auroral arcs (2, 16, $26,76)$, and may actually produce most of the light intensity observed in the aurorae. Some runaways may also become part of the trapped radiation belts. 
- Numerous observations indicate that rapid electron acceleration occurs throughout the outer and auroral zones, especially during substorms and magnetic storms. The possible relationship between the production of relativistic electrons in the earth's magnetosphere and the origin of cosmic rays and the Jovian synchrotron electrons suggests that plasma acceleration processes are universal.

-An accurate model of the inner and outer zone electron fluxes requires that inward radial diffusion be coupled with local electron precipitation losees. The location of the plasmapause and the internal convection and electrons acceleration rates probably specify the initial conditions. During large magnetic storms the inward shift of the plasmapause to $L \sim 2$ permits electron injection directly into the inner zone.

-Shortly, the first space probes will transit the Jovian magnetosphere. From radio emission suudies we know that Jupiter has an inner radiation zone containing intense fluxes of relativistic electrons $(13,75)$. In situ surveys of the entire Jovian radiation environment will help elucidate whether the physics of the earth's magnetosphere is applicable to planetary magnetospheres in general or whether new or perhaps overlooked physical processes must be considered.

\section{Acknowledgments}

It is a pleasure to acknowledge many beneficial discussions with Professor C.F. Kennel and Dr. L.R. Lyons. This work was supported in part by NASA grant NGR-05-007-190, NSF grants GA-2805 and GA-34148. 


\section{REFERENCES}

1. Akasofu, S.I., Polar and Magnetospheric Substorms, Springer, New York, 1969.

2. Albert, R.D., Energy and flux variations of nearly monoenergetic auroral electrons, J. Geophys. Res. 72, 5811, 1967 .

3. Alfven, H. and C.G. Falthammer, Cosmical Electrodynamics, Oxford, Clarendon Press, 1963.

4. Andronov, A.A. and V V. Trakhtengehrts, Kinetic instability of the earth's outer radiation belts, Geomagnet. Aeron. 4, 181, 1964.

5. Axford, W.I., H.E. Petschek and G.L. Siscoe, Tail of the magnetosphere, J. Geophys. Res. 70, 1231, 1965.

6. Bame, S.J., J.R. Asbridge, H.E. Felthauser, E.W. Hones and I.B. Strong, Strong electrons in the plasma sheet in the earth's magnetic tail, Phys. Rev. Letters $16,138,1966$.

7. Birmingham, T.J., Convection electric fields and the diffusion of trapped magnetospheric radiation, J. Geophys. Res. 74, $2169,1969$.

8. Block, L., On the distribution of electric fields in the magnetosphere, J. Geophys. Res. 71, 855, 1966.

10. Burtis, W.J. and R.A. Helliwe11, Banded chorus--a new type of VLF radiation observed in the magnetosphere by 0GO-1 and OGO-3, J. Geophys. Res. 74, 3002, 1969.

11. Carpenter, D.L., Recent research on the magnetospheric plasmapause, Radio Sci. 3, $719,1968$.

12. Carpenter, D.L., K. Stone, J.C. Siren and T. Crystal, Magnetospheric electric fields deduced from drifting whistler paths, J. Geophys. Res. 77, 2819, 1972.

13. Carr, T. and S. Gulkis, The magnetosphere of Jupiter, Ann. Rev. Astron. Astrophys. $7,577,1969$.

14. Cauffman, D.P. and D.A. Gurnett, Double-probe measurements of convection electric fields with the Injun-5 satellite, J. Geophys. Res. 76, 6014, 1971.

15. Chappe11, C.R., D.D. Harris and G.W. Sharp, OGO-5 measurements of the plasmasphere during observations of stable auroral red arcs, J. Geophys. Res. 76, 2357, 1971.

16. Chase, L.M., Energy spectra of auroral zone particles, J. Geophys. Res. 75, 7128,1970 .

17. Cornwa11, J.M., Micropulsations and the outer radiation zone, J. Geophys. Res. 71, $2185,1966$.

18. Cornwall, J.M., Diffusion processes influenced by conjugate-point wave phenomena, Radio Sci. 3, 740, 1968.

19. Cornwall, J.M., F.V. Coroniti and R.M. Thorne, Turbulent loss of ring current protons, J. Geophys. Res. 75, 4699, 1970.

20. Cornwal1, J.M., Radial diffusion of ionized helium and protons: a probe for magnetospheric dynamics, J. Geophys. Res. 77, 1756-1770, 1972.

21. Coroniti, F.V. and C.F. Kennel, Polarization of the auroral electrojet, J. Geophys. Res. $77,2835,1972$.

22. Coroniti, F.V. and C.F. Kennel, Changes in magnetospheric configuration during substorm growth phase, J. Geophys. Res. 77, , 1972.

23. Craven, J.D., Temporal variations of electron intensities in the outer radiation zone as observed with satellite Injun 3, J. Geophys. Res. 71, 5643, 1966.

24. DeForest, L.A. and C.E. McIlwain, Plasma clouds in the magnetosphere, J. Geophys. Res. 76, 3587, 1971 .

25. Dungey, J.W., Interplanetary magnetic field and the auroral zones, Phys. Rev. Letters 6, 47, 1961.

26. Evans, D.S., The observations of a near monoenergetic flux of auroral electrons, J. Geophys. Res. 73, 2315, 1968.

27. Falthammer, C.G., Effects of time-dependent electric fields on geomagnetically trapped radiation, J. Geophys. Res. 70, 2503, 1963.

28. Forbush, S.E., D. Venkateswaran and C.E. McIlwain, Intensity variations in outer Van Allen radiation belt, J. Geophys. Res. 66, 2275, 1961 .

29. Forbush, S.E., G. Pizzella and D. Venkateswaran, The morphology and temporal variations of the Van Allen radiation belt, October 1959 to December 1960, J. Geophys. Res. 67, 3651, 1962 . 
30. Frank, L.A., J.A. Van Allen and H.K. Hills, A study of charged particles in the earth's outer radiation zone with Explorer 14, J. Geophys. Res. 69, 2171, 1964.

31. Frank, L.A., Explorer 12 observations of the temporal variations of low-energy electron intensities in the outer radiation zone during geomagnetic storms, J. Geophys. Res. 71, 4631, 1966.

32. Frank, L.A. Several observations of low-energy protons and electrons in the earth's magnetosphere with OGO-3, J. Geophys. Res. 72, 1905-1916, 1967a.

33. Frank, L.A. and K.L. Ackerson, Observations of charged particle precipitation into the auroral zone; J. Geophys. Res. 76, 3612, 1971.

34. Frank, L.A., Plasma in the earth's polar magnetosphere, J. Geophys. Res. 76, $5202,1971$.

35. Gurnett, D.A. and L.A. Frank, Observed relationships between electric fields and auroral particle precipitation, J. Geophys. Res. 77, , 1972.

36. Haerende1, G., On the balance between radial and pitch angle diffusion, in Particles and Fields in the Magnetosphere, ed. by B.M. McCormac, 416, D. Reide1, Dordrecht, Holland, 1970.

37. Heikkila, W.J. and J.D. Winningham, Penetration of magnetosheath plasma to low altitudes through the dayside magnetospheric cusps, J. Geophys. Res. 76, 883,1971 .

38. Hones, E.W., S. Singer and C.S.R. Rao, Simultaneous observations of electrons $(E>45 \mathrm{KeV})$ at $2000 \mathrm{kilometers} \mathrm{altitude}$ and at 100,000 kilometers in the magnetotail, J. Geophys. Res. 73, 7330, 1968.

39. Hones, E.W., Jr., J.R. Asbridge, S.J. Bame and S. Singer, Energy spectra and angular distributions of particles in the plasma sheet and their comparison wihh rocket measurements over the auroral zone, J. Geophys. Res. 76, 63, 1971 .

40. Kavanagh, L.D., J.W. Freeman, and A.J. Chen, plasma flow in the magnetosphere, J. Geophys. Res. 73, 5511, 1968.

41. Kennel, C.F. and H.E. Petschek, A limit on stably trapped particle fluxes, J. Geophys. Res. 71, 1, 1966.

42. Kennel, C.F. and H.E. Petschek, Van Allen belt plasma physics, in Proceedings Second Orsay Summer Institute on Plasma Physics, Kalman and Feix, eds., 95, Gordon and Breach, London, 1969.

43. Kennel, C.F., Consequences of a magnetospheric plasma, Rev. of Geophysics 7; 379,1969 .

44. Kenne1, C.F., F.L. Scarf, R.W. Fredricks, J.H. McGehee and F.V. Coroniti, VLF electric field observations in the magnetosphere, J. Geophys. Res. 35, 6136,1970 .

45. Kindel, J.M. and C.F. Kennel, Topside current instabilities, J. Geophys. Res. 76, $3055,1971$.

46. Laval, G. and R. Pellat, Particle acceleration by electrostatic waves propagating in an inhomogeneous plasma, J. Geophys. Res. 75, 3255, 1970.

47. Levy, R.H., H.E. Petschek and G.L. Siscoe, Aerodynamic aspects of the magnetospheric flow, AIAAJ 2, 2065, 1964.

48. Lyons, L.R. and R.M. Thorne, The magnetospheric reflection of whistlers, Planet. Space Sci. 18, 1753, 1970 .

49. Lyons, L.R., R.M. Thorne and C.F. Kennel, Electron pitch-angle diffusion driven by oblique whistler mode turbulence, J. Plasma Physics 6, 589, 1971.

50. Lyons, L.R., R.M. Thorne and C.F. Kennel, Pitch-angle diffusion of radiation belt electrons within the plasmasphere, J. Geophys. Res. 77, , 1972.

51. McIlwain, C.E., Coordinates for mapping the distribution of magnetically trapped particles, J. Geophys. Res, 66, 3681, 1961.

52. Mozer, F.S., Power, spectra of the magnetospheric electric field, J. Geophys. Res. $76,3651,1971$.

53. Muzzio, J.L.R. and J.J. Angerami, OGO-4 observations of extremely low frequency hiss, J. Geophys. Res. 77, 1157, 1972.

54. Northrup, T.G., The adiabatic motion of charged particles, Interscience Publishers, 1963.

55. Owens, H.D. and L.A. Frank, Electron omnidirectional intensity contour's in the earth's outer radiation zone at the magnetic equator, J. Geophys. Res. 73, $199,1968$. 
56. Parks, G.K. and J.R. Winckler, Acceleration of energetic electrons observed at the synchronous altitude during magnetospheric substorms, J. Geophys. Res. $73,5786,1968$.

57. Petschek, H.E., The mechanism for reconnection of geomagnetic and interplanetary field lines, in The Solar Wind, ed. by R.J. Mackin and M. Neugebauer, 257, Pergamon Press, New York, 1966.

58. Petschek, H.E. and C.F. Kenne1, Tail flow, auroral precipitation, and ring currents, Trans. Amer. Geophys. Union 47, 137, 1966.

59. Pfitzer, K.A., S. Kane and J.R. Winckler, The spectra and intensity of electrons in the radiation belts, Space Res. 6, 702, 1966.

60. Pfitzer, K.A. and J.R. Winckler, Experimental observation of a large addition to the electron inner radiation belt after a solar flare event, $J$. Geophys. Res. 73, 5792, 1968.

61. Roederer, J.G., Dynamics of Geomagnetically Trapped Radiation, Springer-Verlag, New York, 1970 .

62. Rothwell, P. and C. Lynam, The plasmapause, the plasma sheet and energetic trapped electrons in the earth's magnetosphere, Planet. Space Sci, 17, $447,1969$.

63. Rosser, W.G.V., Changes in the structure of the outer radiation zone associated with the magnetic storm of September 30, 1961, J. Geophys. Res. 68,3131, 1963.

64. Russe11, C.T. and R.M. Thorne, On the structure of the inner magnetosphere, Cosmic Electrodynamics $1,67,1970$.

65. Schield, M.A. and L.A. Frank, Electron observations between the inner edge of the plasma sheet and the plasmasphere, J. Geophys. Res. 75, 5401, 1970.

66: Swift, D.W., Particle acceleration by electrostatic waves, J. Geophys. Res. 75, $6324,1970$.

67. Taylor, H.E. and E.W. Hones, Adiabatic motion of auroral particles in a model of the electric and magnetic fields surrounding the earth, J. Geophys. Res. 70; 3605,1965 .

68. Throne, R.M. and C.F. Kennel, Relativistic electron precipitation during magnetic storm main phase, J. Geophys. Res. 76, 4446, 1971.

69. Throne, R.M., ELJL Smith, R.K. Burton and R.E. Holzer, Plasmaspheric hiss, J. Geophys. Res. 77, , 1972.

70. Tomassian, A.D., T.A. Farley and A.L. Vampola, Inner zone energetic electron repopulation by radial diffusion, J. geophys. Res. 77, , 1972.

71. Vampola, A.L., Electron pitch-angle scattering in the outer zone during magnetically disturbed times, letter to J. Geophys. Res. 76, 4685, 1971.

72. Vasyliunas, V.M., Low-energy electrons on the dayside of the magnetosphere, J. Geophys. Res. 73, 7519, 1968.

73. Vasyliunas, V.M., A survey of low energy electrons in the evening sector of the magnetosphere with OGO -1. and OGO-3, J. Geophys. Res $\therefore$ 73, 2839, 1969.

74. Vernov, S.N., E.V. Gorchakov, S.N. Kuznetsov; Yu.I. Logachev, E.N. Sosnovets and V.G. Stolpovsky, Particle fluxes in the outer geomagnetic field, Rev. of Geophys. 7, 257, 1969.

75. Warwick, J.W., Radiophysics of Jupiter, Space Sci. Rev. 6, 841, 1967.

76. Westerlund, L.H., The auroral electron energy spectrum extended to $45 \mathrm{eV}$, J. Geophys. Res. 74, 351, 1969.

77. Williams, D.J., J.F. Arens and L.J. Lanzerotti, Observations of trapped electrons at low and high altitudes, J. Geophys. Res., 73, 5673, 1968.

78. Winckler, J.R., The origin and distribution of energetic electrons in the Van Allen radiation belts, in Particles and Fields in the Magnetosphere, ed. B.M. McCormac, Reidel Pub1. Co., Dordrecht, Holland, 1970 .

79. Young, T.S.T., J.D. Callen and J.E. McCune, High frequency electrostatic waves in the magnetosphere, J. Geophys. Res. 77, , 1972. 
TABLE I: $50 \mathrm{eV}$ to $50 \mathrm{KeV}$ Spectral Integrals

(From S.E. DeForest and C.E. McIlwain, 24)

\begin{tabular}{|c|c|c|c|c|c|c|}
\hline \multirow[t]{2}{*}{. } & \multicolumn{6}{|c|}{ Electrons } \\
\hline & $\begin{array}{l}0000 \\
\text { LT }\end{array}$ & $\begin{array}{l}0300 \\
\text { LT }\end{array}$ & $\begin{array}{c}0600 \\
\text { LT }\end{array}$ & $\begin{array}{l}1200 \\
\mathrm{LT}\end{array}$ & $\begin{array}{l}1800 \\
\text { LT }\end{array}$ & $\begin{array}{c}2100 \\
\text { LTT }\end{array}$ \\
\hline \multicolumn{5}{|c|}{ Number Density, particles $/ \mathrm{cm}^{3}$} & & . \\
\hline Minimum & 0.07 & 0.22 & 0.07 & 0.06 & 0.02 & 0.04 \\
\hline Maximum & 8.3 & 4.8 & 2.9 & 1.2 & 1.9 & 4.4 \\
\hline Typical & 2.0 & 2.0 & 1.2 & 0.4 & 0.10 & 0.4 \\
\hline \multicolumn{7}{|c|}{ Energy $\mathrm{flux}, \mathrm{erg} / \mathrm{cm}^{2} \sec$ ster } \\
\hline Minimum & 0.21 & 0.38 & 0.42 & 0.26 & 0.04 & 0.10 \\
\hline Maximum & 9.4 & 15.2 & 14.6 & 2.3 & 1.01 & 7.2 \\
\hline Typical & 3.0 & 3.0 & 1.5 & 1.0 & 0.40 & 0.5 \\
\hline \multicolumn{5}{|c|}{ Number flux, $10^{6}$ particles $/ \mathrm{cm}^{2}$ sec ster } & & . \\
\hline Minimum & 15 & 37 & 32 & 15 & 2 & 9 \\
\hline Maximum & 1510 & 1020 & 832 & 122 & 162 & 864 \\
\hline Typical & 300 & 300 & 200 & 70 & 30 & 60 \\
\hline \multicolumn{7}{|c|}{ Pressure, $10^{-10}$ dynes $/ \mathrm{cm}^{2}$} \\
\hline Minimum & 2.7 & 6 & 6 & 4 & 0.4 & 1 \\
\hline Maximum & 190 & 266 & 173 & 25 & 14 & 128 \\
\hline Typical & 50 & 60 & 30 & 12 & 7 & 8 \\
\hline
\end{tabular}




\section{FIGURE CAPTIONS}

Figure 1: The magnetosphere's magnetic field topology and plasma environment. Lines of force emanating from the polar caps are connected to interplanetary field lines.

Figure 2a,b: Quiet (left) and substorm (right) differential electron energy spectra. The dashed curve is for a Maxwellian distribution with e-folding energy $\mathrm{E}_{0}$. (After Hones et al, 39).

Figure 3: Differential auroral electron energy spectra. Curve $I$ is a pre-substorm and curve II is a post-substorm spectra detected on the geostationary satellite ATS- 5 by DeForest and McIlwain (24). Curve III is a spectrum taken inside a inverted-V event on Injun $V$ by Frank and Ackerson (33). An inverted-V is characterized by a spatial increase of the mean electron energy followed by a decrease. Curve IV is taken inside an auroral arc by Westerlund (76). In curves III and IV the electrons below $50 \mathrm{eV}$ are probably secondary electrons produced by the more energetic primary precipitation.

Figure 4: On the left are shown the electron drift trajectories and constant energy contours (in $\mathrm{KeV}$ ) for electrons which start at $10 \mathrm{R}_{\mathrm{E}}$ on the nightside with $1 \mathrm{KeV}$ energy in a $2 \times 10^{-4}$ Gauss field. The dots represent the electron position at 10 minute intervals. (After Kavanagh et al., 40). On the right is shown the electron drift paths (arrowed curves) for an initial flow velocity in the tail of $15 \mathrm{~km} / \mathrm{sec}$ and an initial temperature of $1 \mathrm{KeV}$. The heavy curves are contours of constant electron temperature. Due to the precipitation of hot plasma sheet electrons and their replacement by thermalized ionospheric electrons, there is a sharp temperature gradient at the plasma sheet inner edge. (After Vasyliunas in Kennel, 43).

Figure 5: Differential electron flux vs. L. Channe1 8 measures electrons within the energy range 0.99 to $1.7 \mathrm{KeV}$, channel 10 between 2.6 and $4.6 \mathrm{KeV}$, channel 12 between 6.8 and $12 \mathrm{KeV}$, channel 14 between 14 and $24 \mathrm{KeV}$. Other channels are intermediate and below these energies. (After Schield and Frank, 65).

Figure 6: Electron fluxes measured by Parks and Winckler (56) on a geosynchronous satellite. Enhancements by factors of 10-100 occur within minutes of a substorm expansion phase indicated by the magnetic bay occurring near local midnight at College.

Figure 7: Electron differential flux measured by Pfitzer, Kane and Winckler (59) during quiet-times (September 20) and during the main phase (September 29) and recovery (October 20 ) of a modest geomagnetic storm.

Figure 8: Contours of constant omnidirectional intensity of electron $\left(\mathrm{E}_{\mathrm{e}}>40 \mathrm{KeV}\right.$, $>230 \mathrm{KeV}$ and $>1.6 \mathrm{MeV}$ ) at the magnetic equator constructed by Owens and Frank (55) for the period February through March, 1963. During geomagnetically active periods enhancements of low energy electron flux occur coincident with dramatic drop-outs in $>1.6 \mathrm{MeV}$ electrons.

Figure 9: Bounce-averaged cyclotron and Landau resonant pitch-angle diffusion coefficients plotted as a function of equatorial pitch angle at $L=4$ for 20, 200 and $2000 \mathrm{KeV}$. electrons. (After Lyons, et a1., 50).

Figure 10: A comparison between theoretical equatorial pitch-angle distributions and those observed within the electron "slot" during the decay following an injection event (West, private communication).

Figure 11: Theoretical precipitation lifetimes as a function of $L$ for several electron energies. The results apply only within the plasmasphere.

(After Lyons et al., 50). 


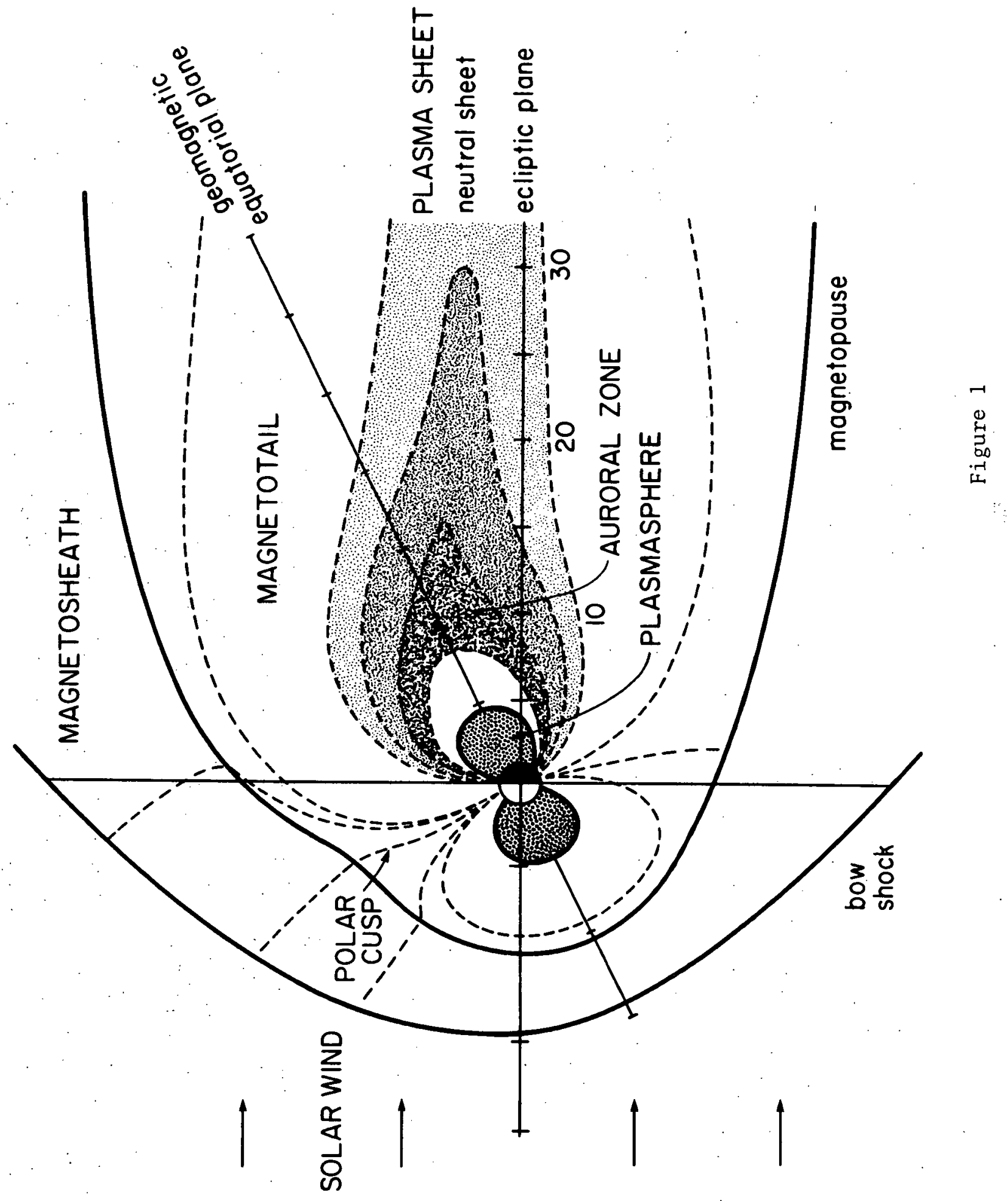




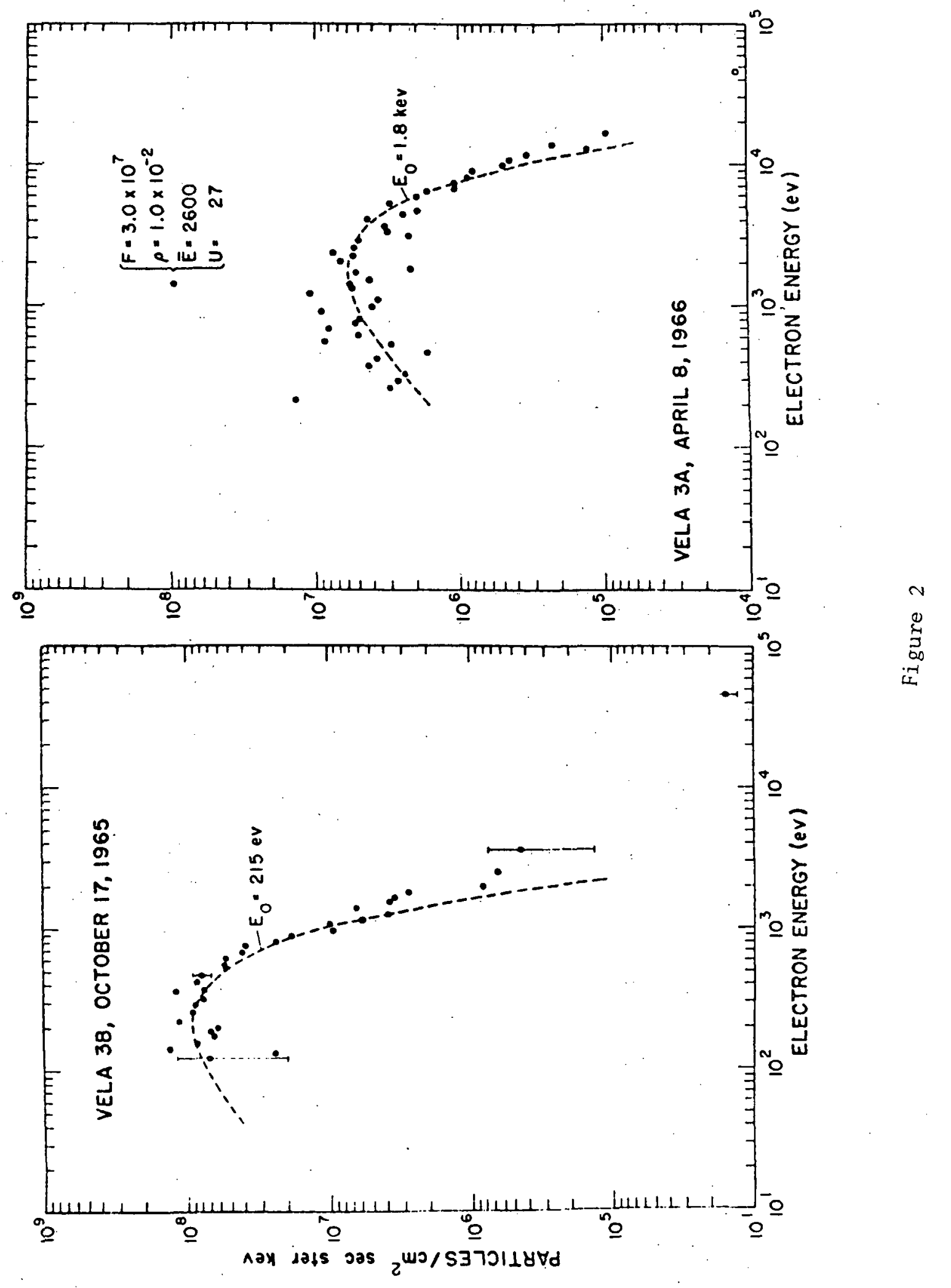




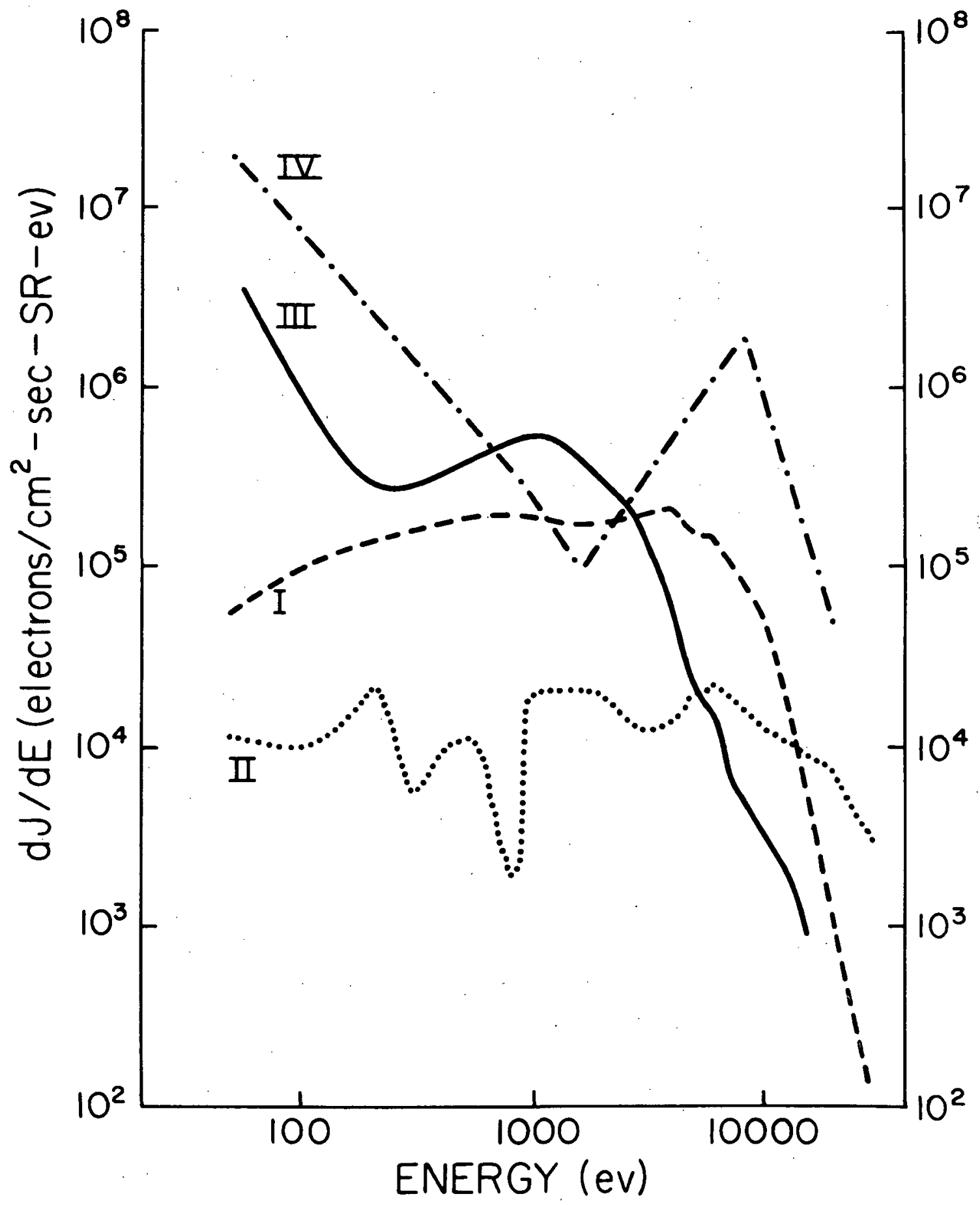

Figure 3 

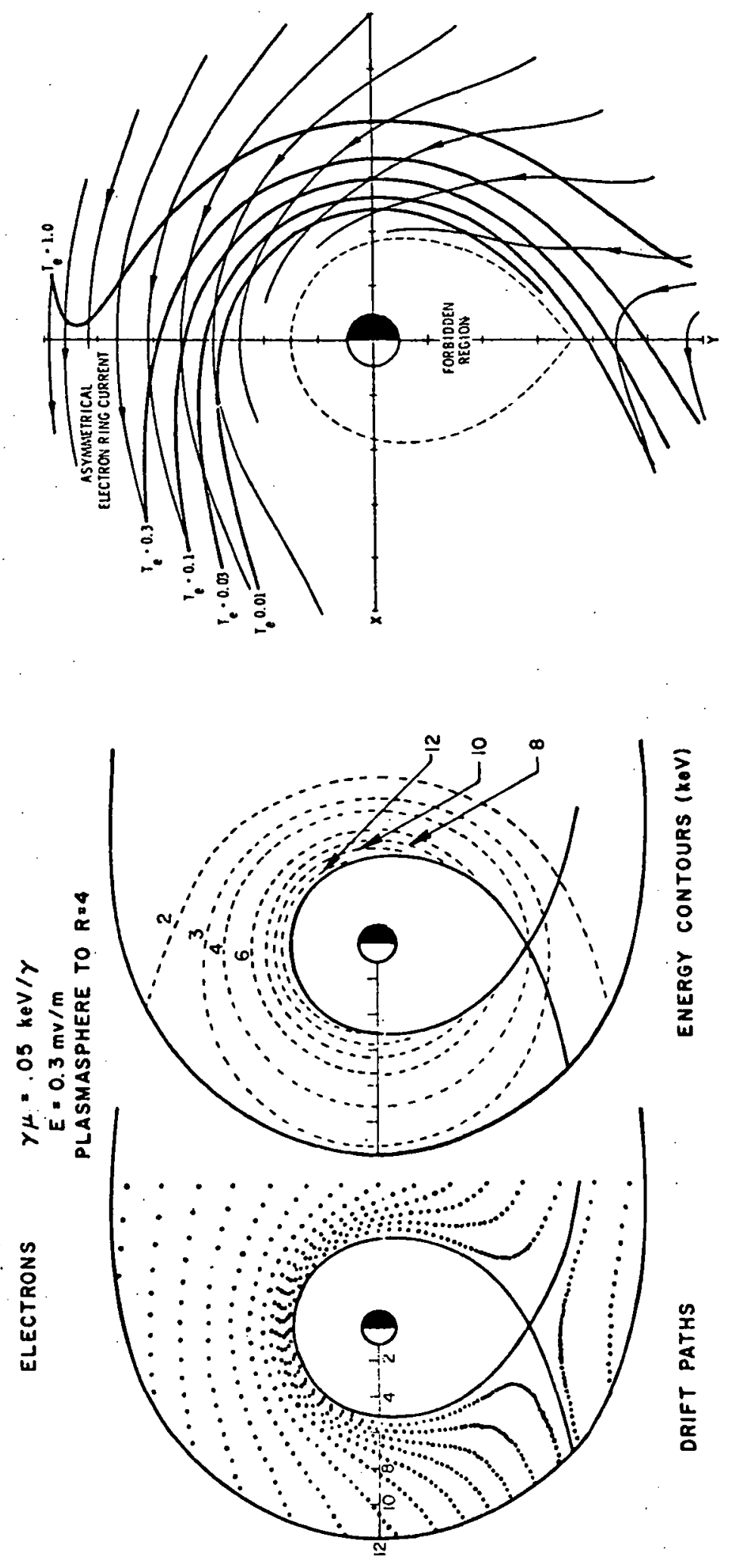

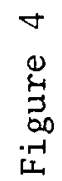




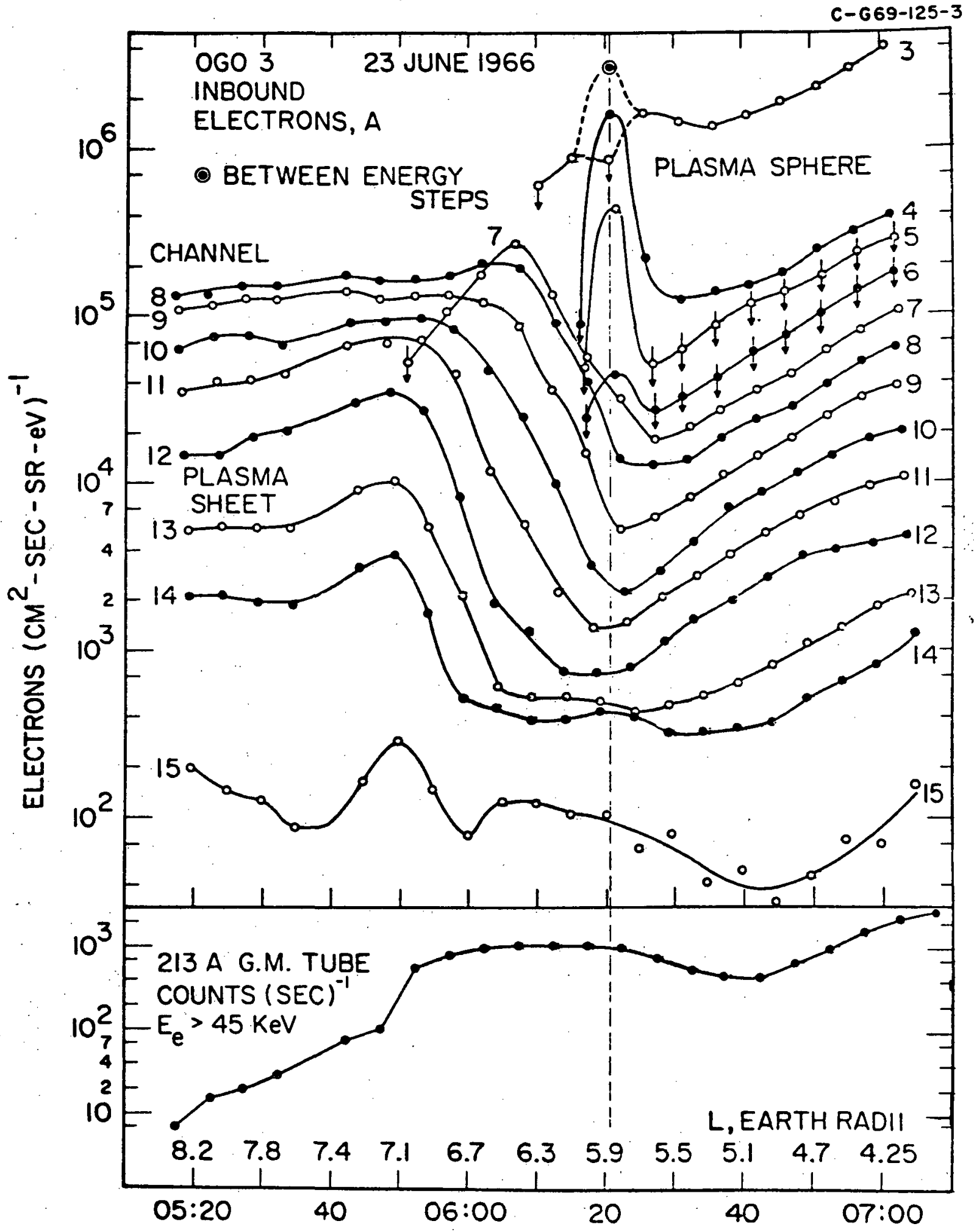

Figure 5 


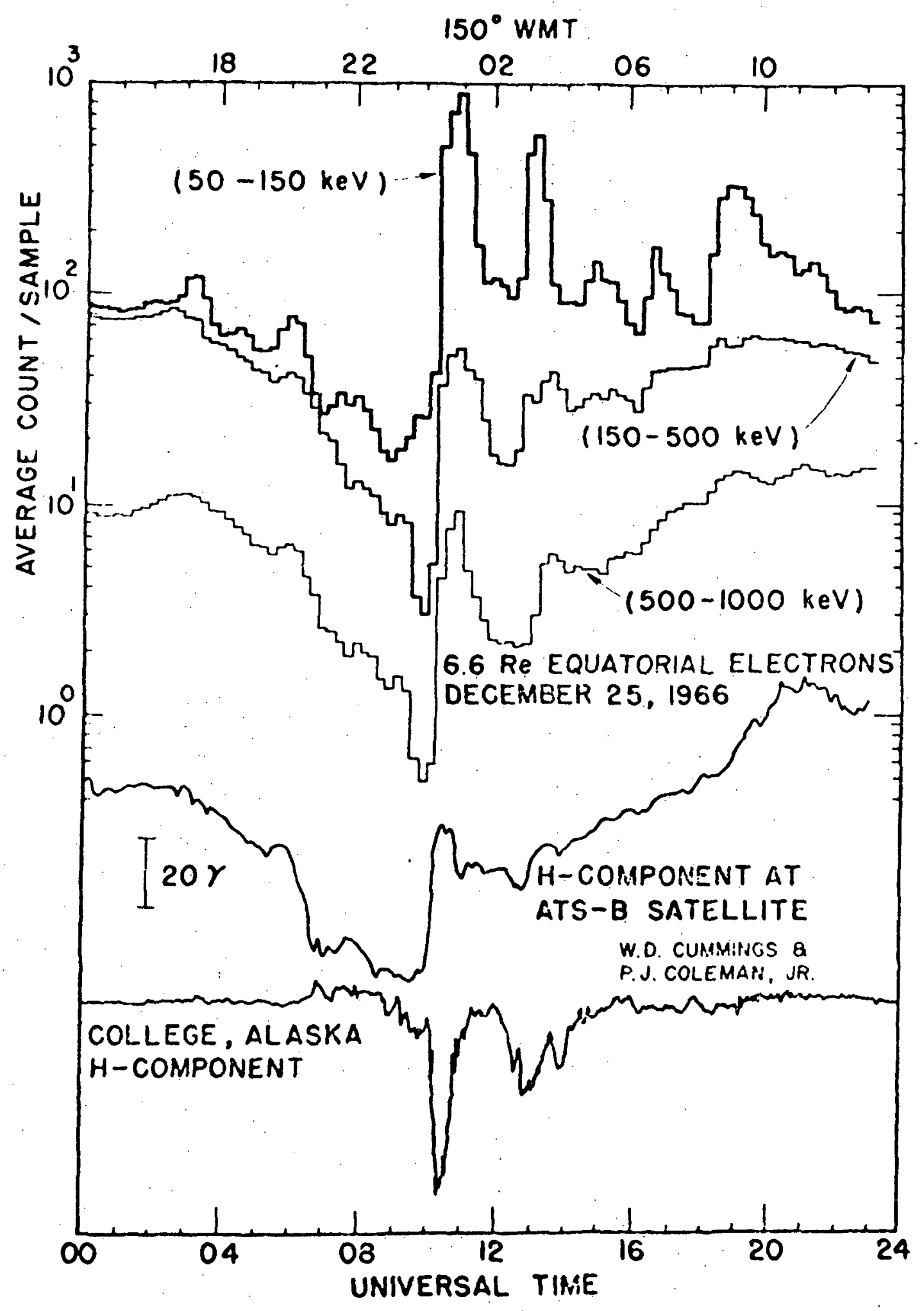

Figure 6 

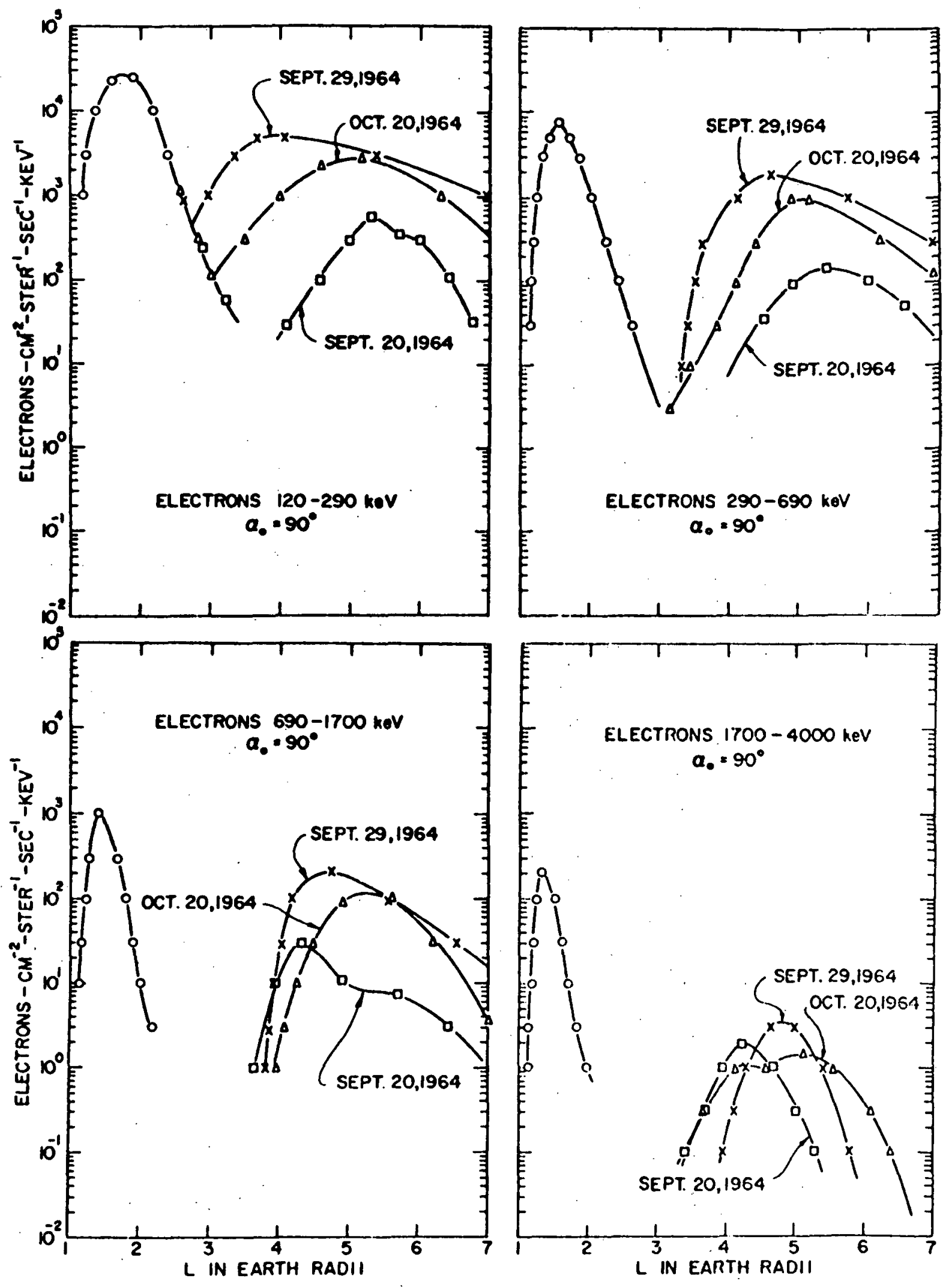


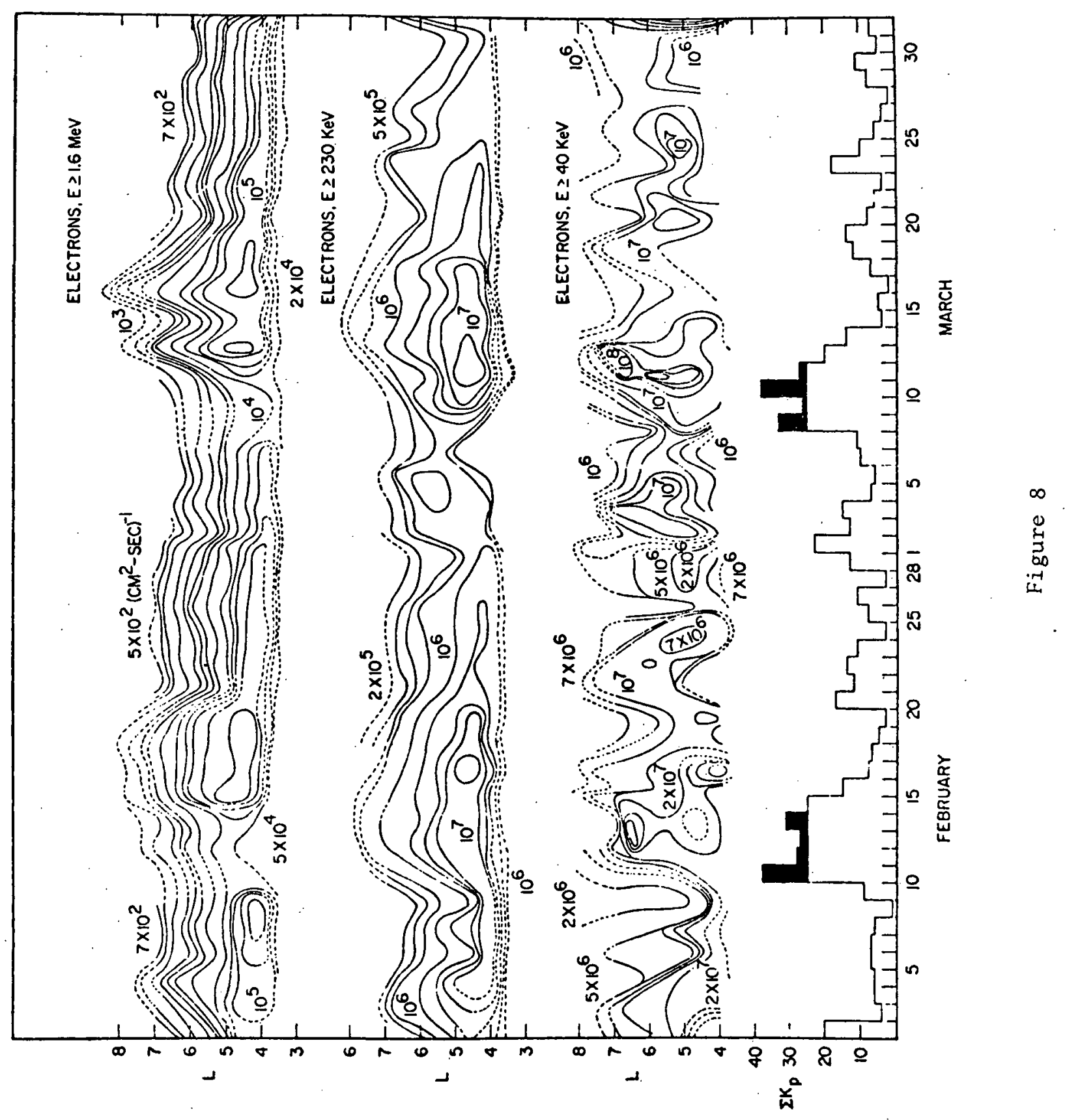




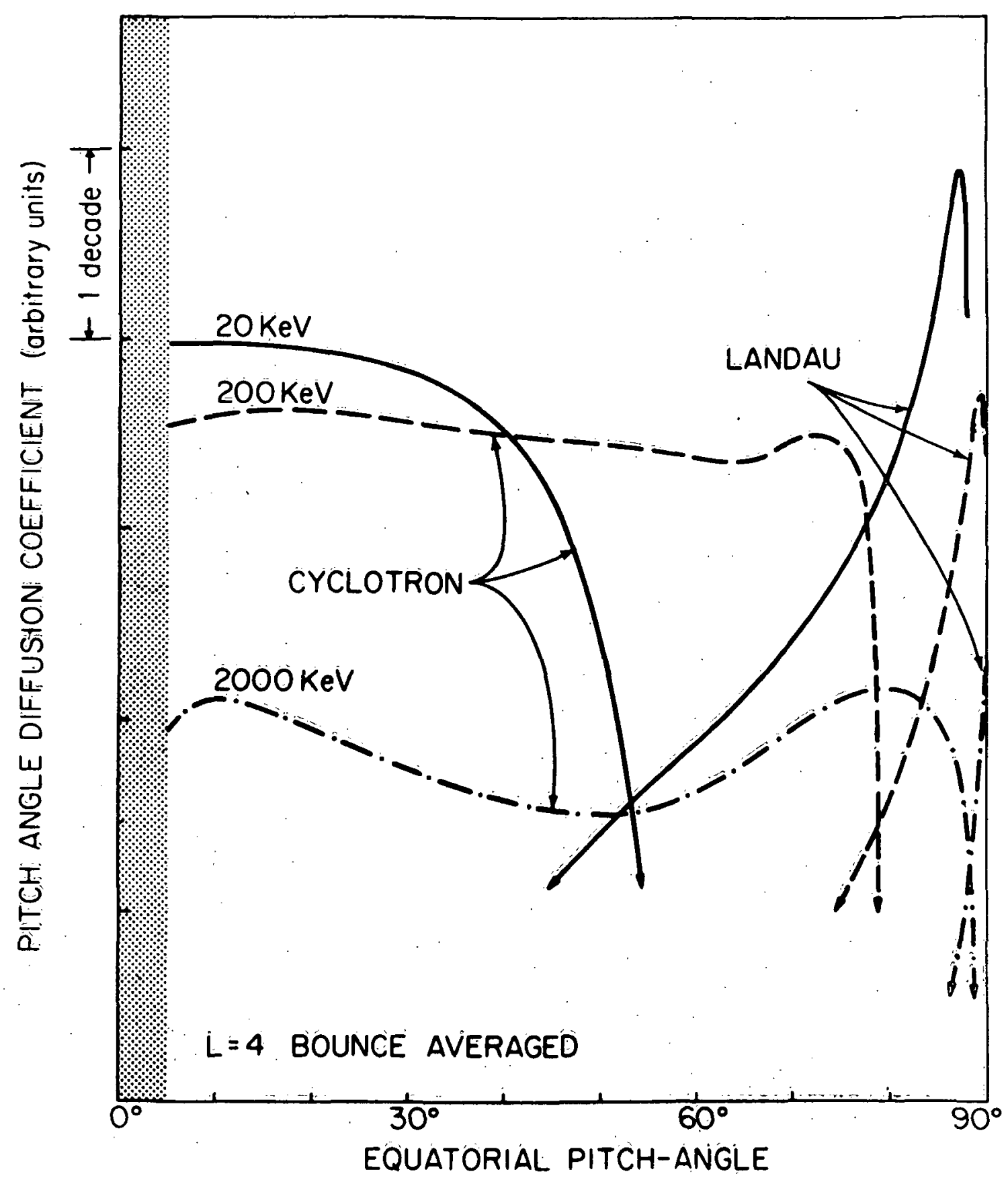

Figure 9 

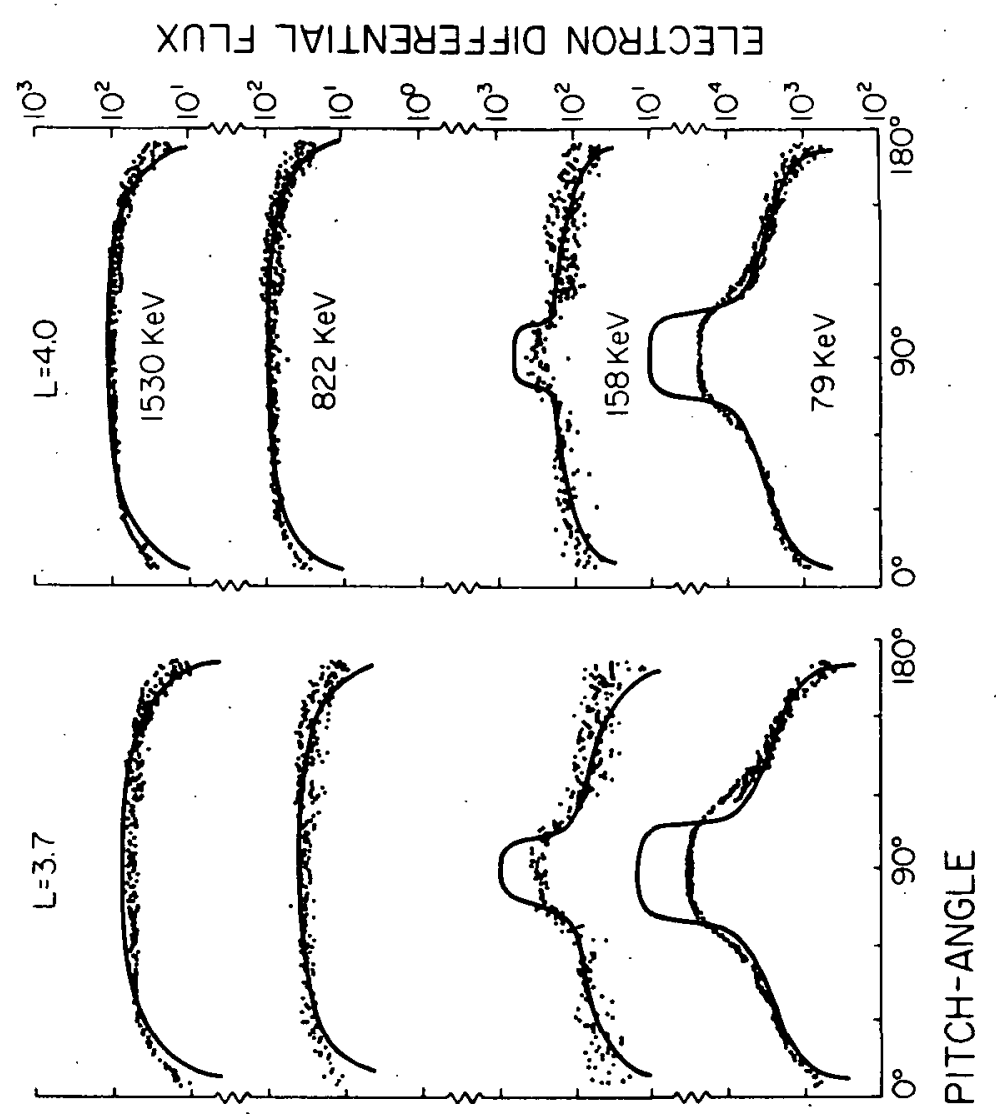

엄
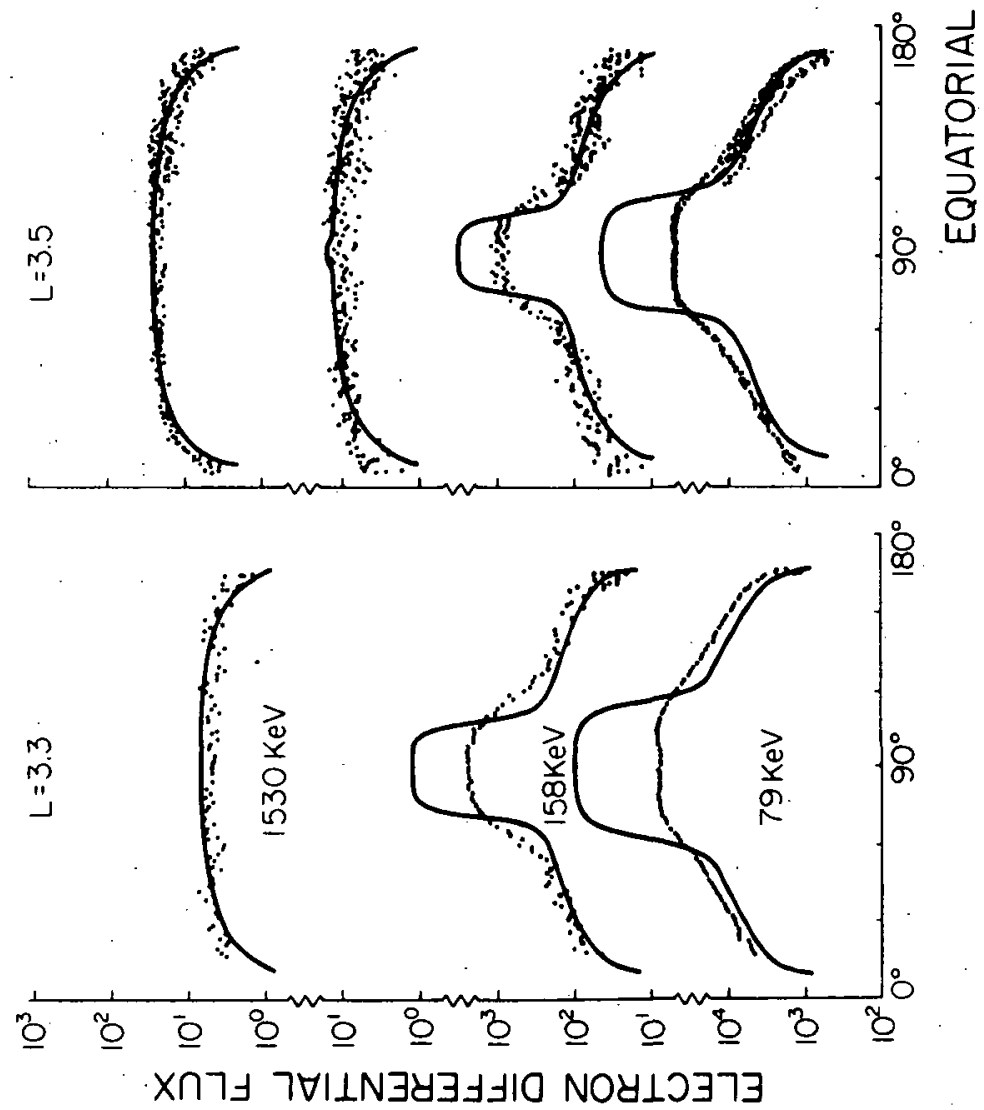


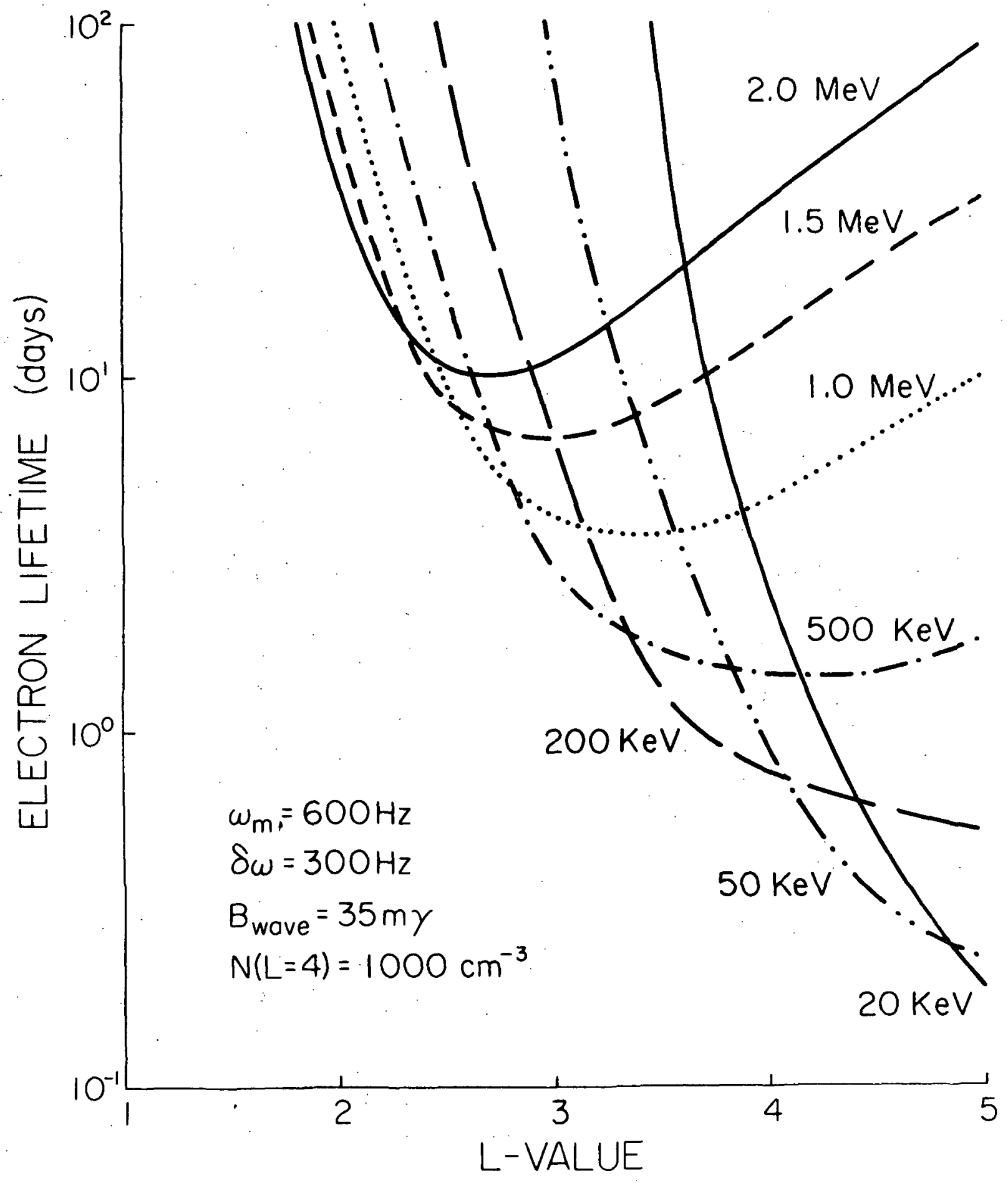

Figure 11 


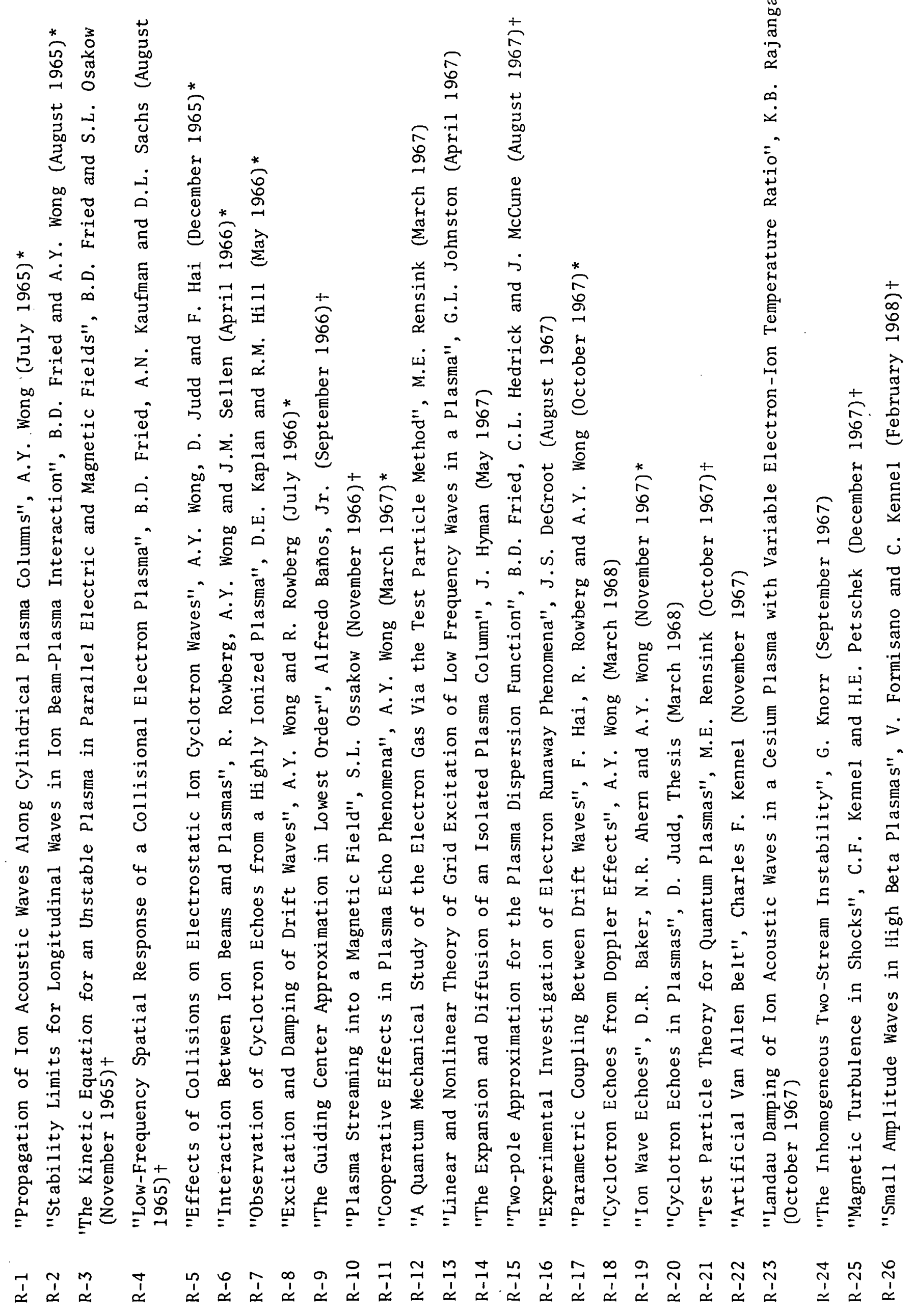




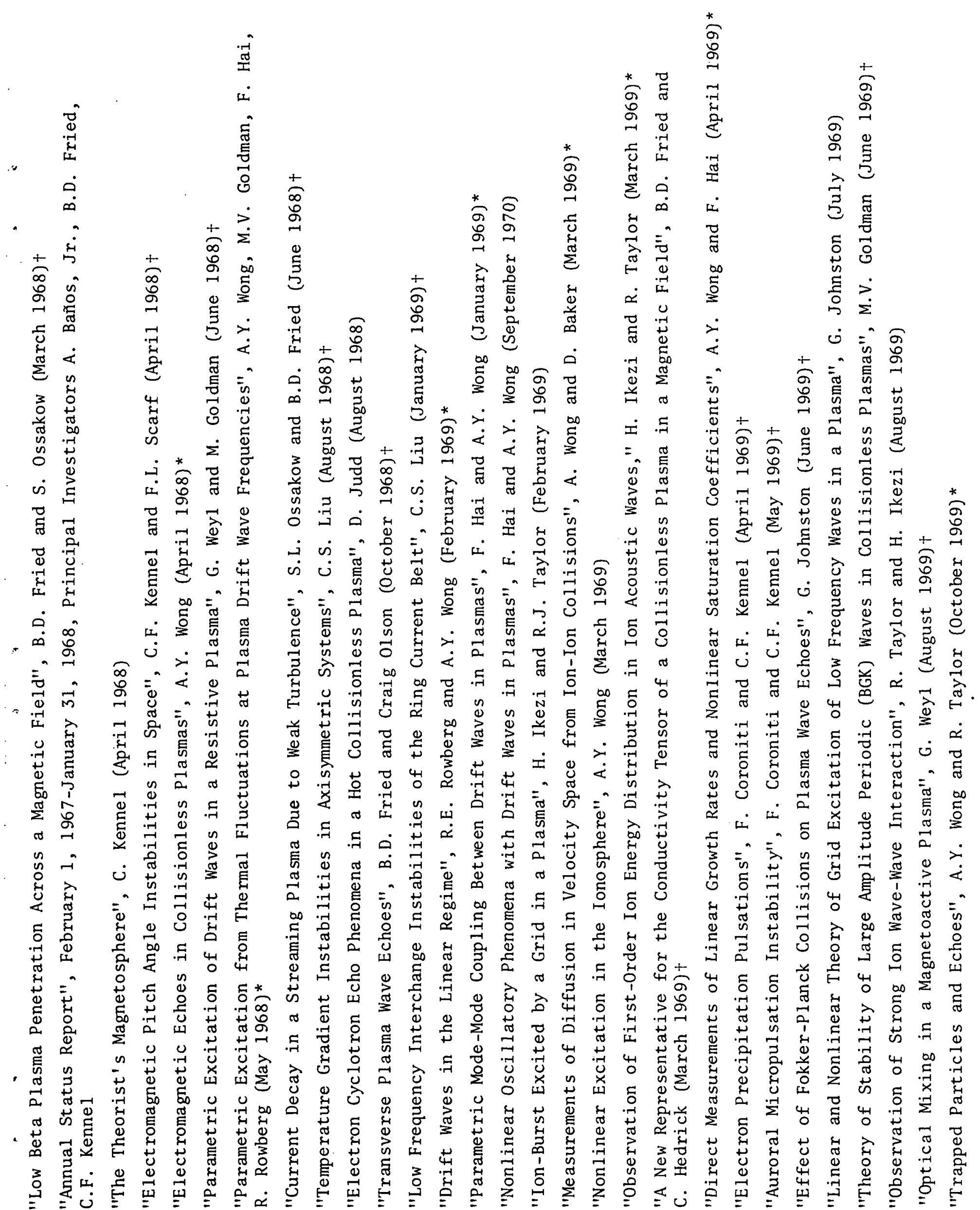

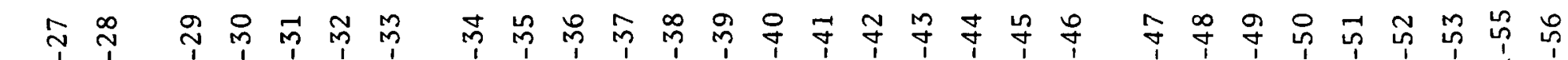

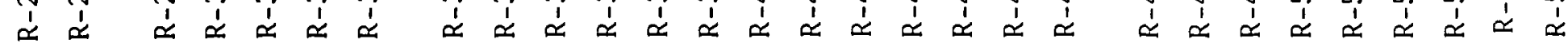




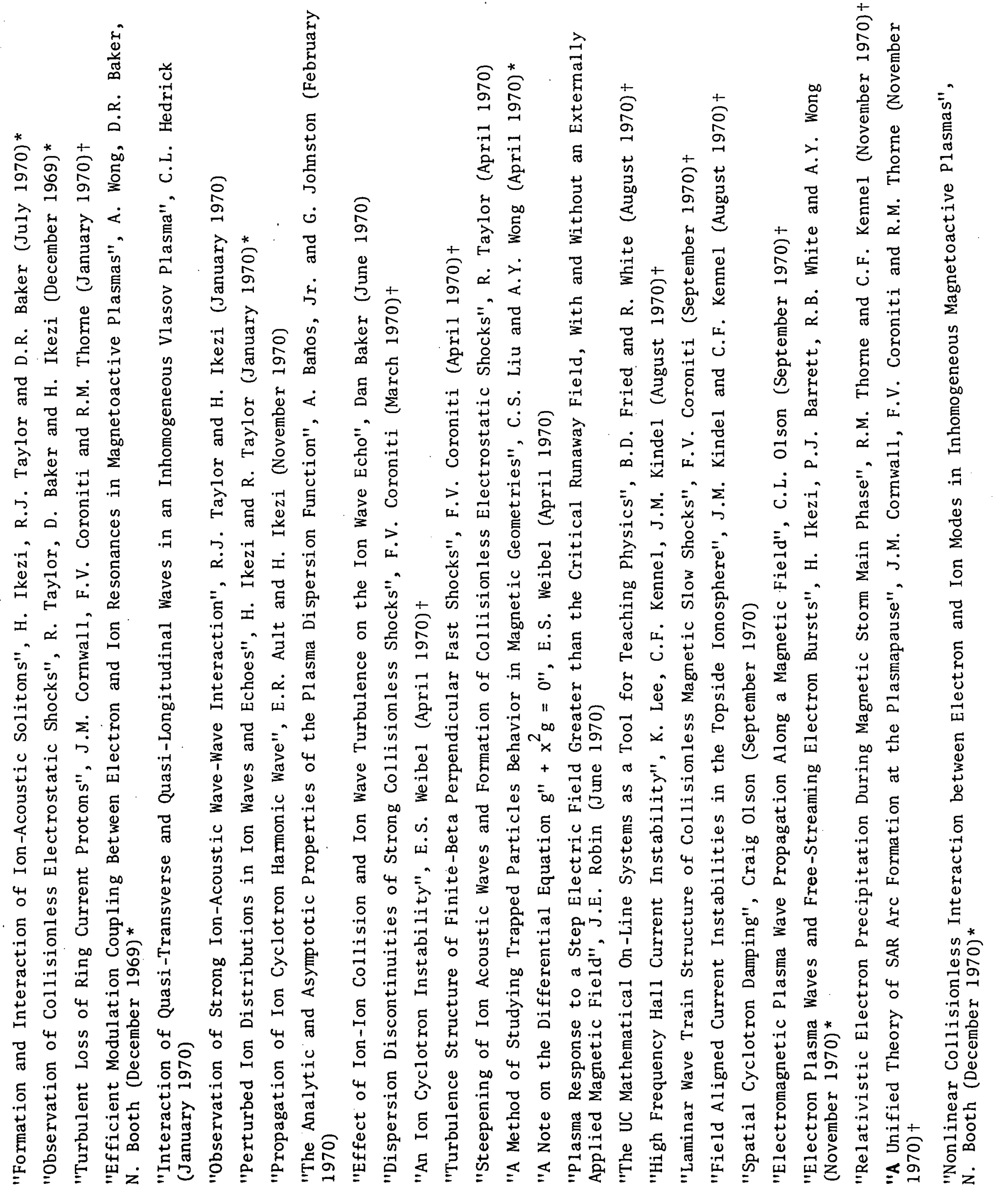




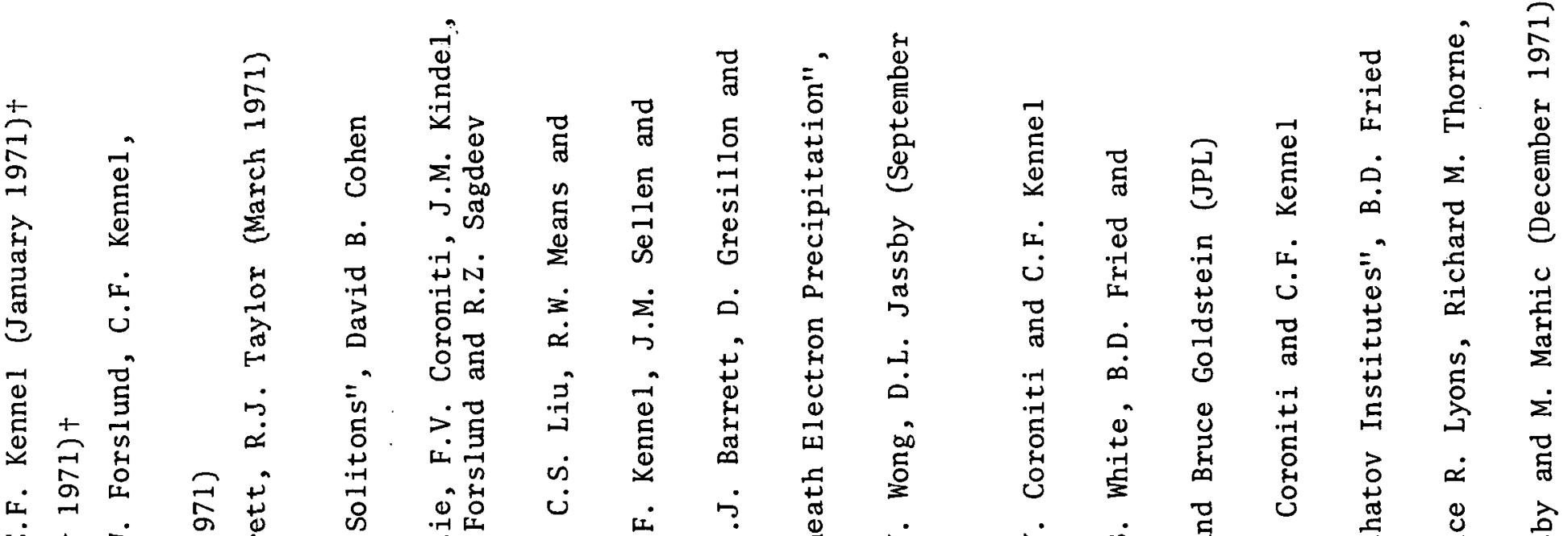

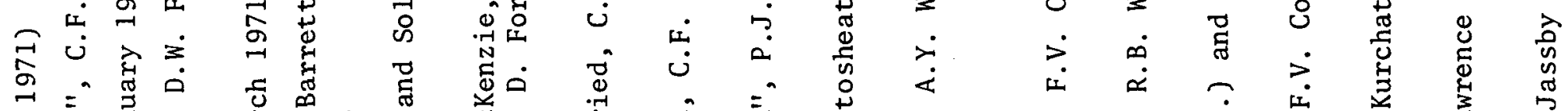

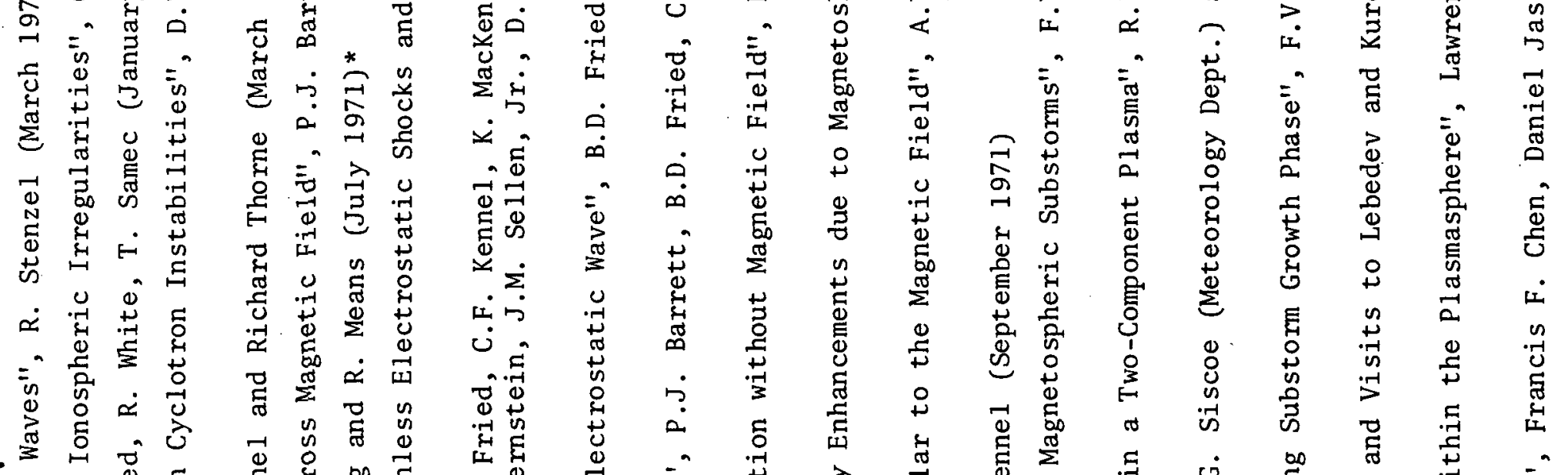

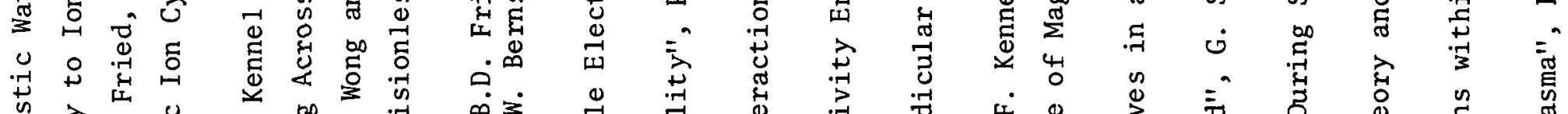

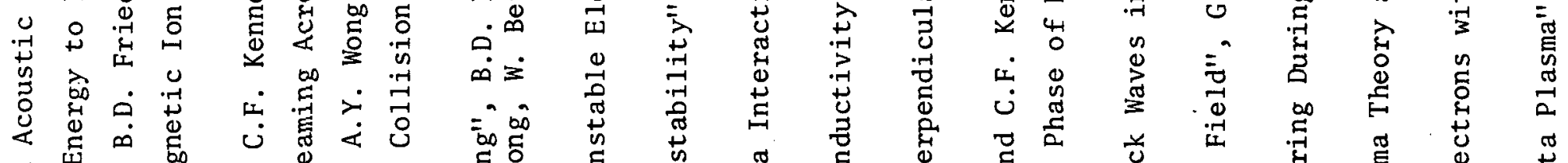

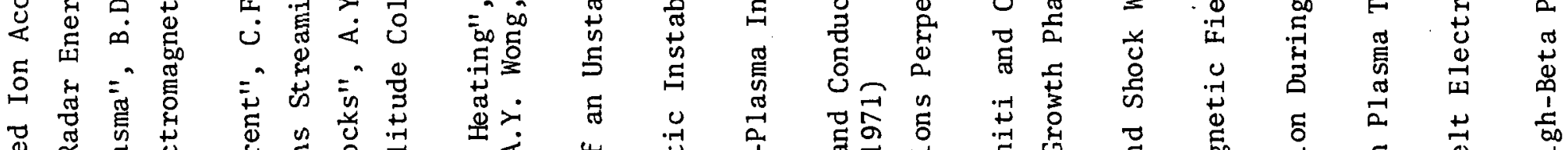

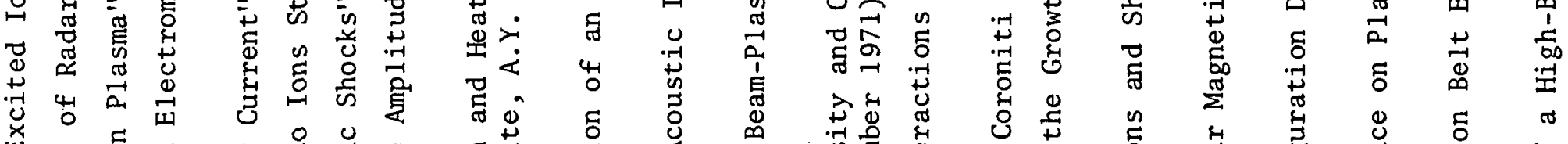

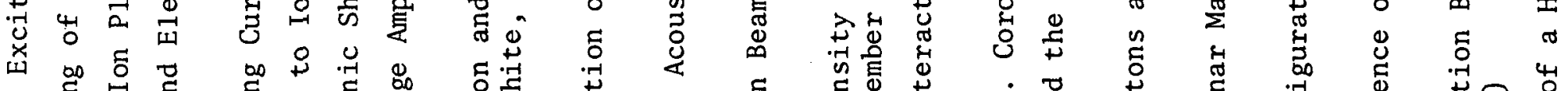

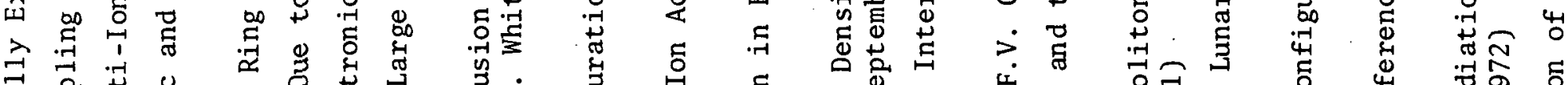
预

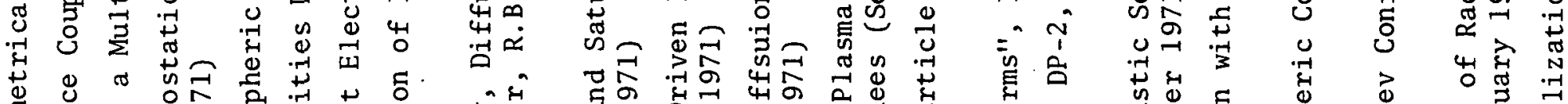

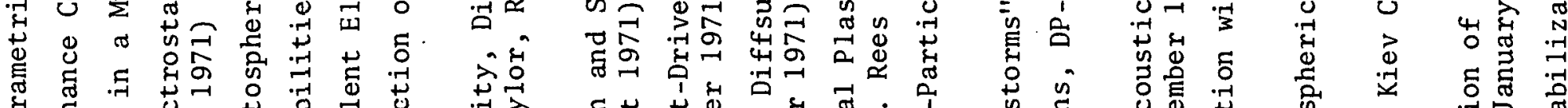

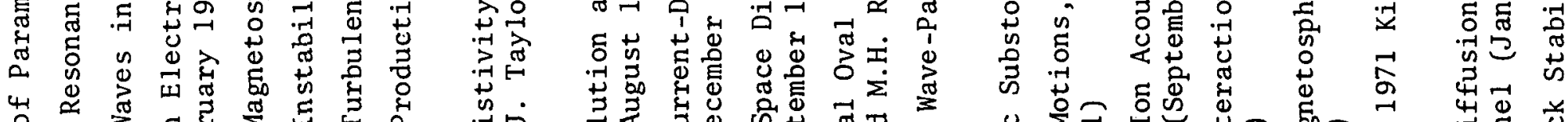

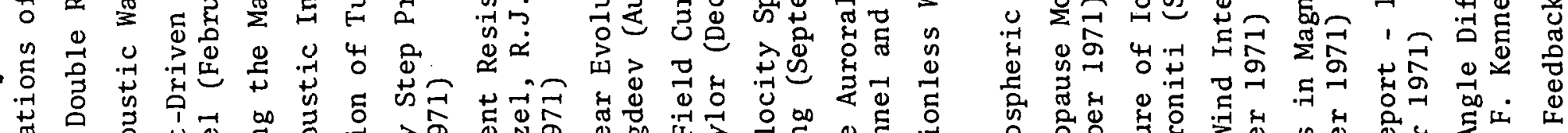

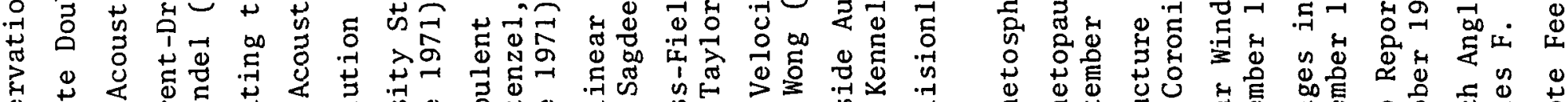

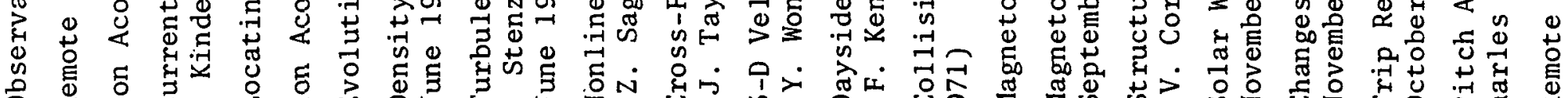
苛

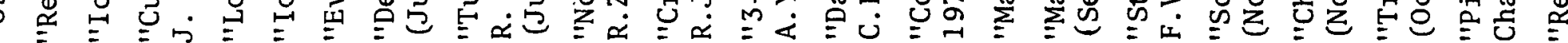

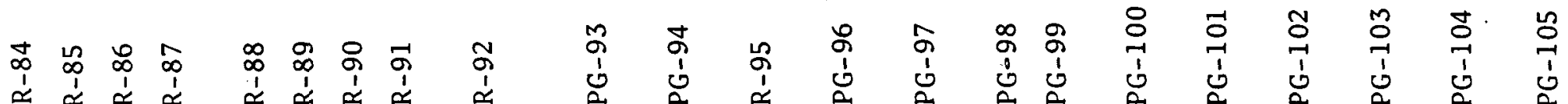

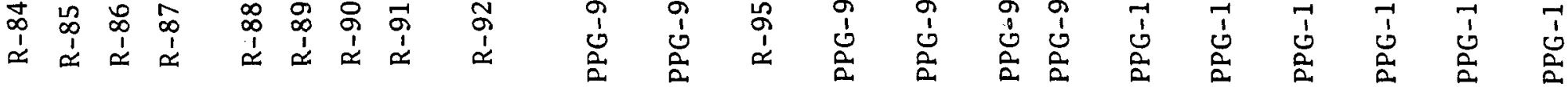

